\title{
Lists of Archeomagnetic and Paleomagnetic Results
}

\author{
-1950-1970, in Japan — \\ H. KINOSHITA* \\ Geophysical Institute, University of Tokyo
}

(Received August 3, 1970)

\begin{abstract}
All the data of paleomagnetic and archeomagnetic studies made in these two decades in Japan are presented as three tables. Two of them give the paleomagnetic and archeomaghetic direction studies and the other table gives results of intensity studies from the recent to the geological past.
\end{abstract}

\section{Introduction}

A vast number of data of the paleomagnetism and the archeomagnetism has recently been accumulated in Japan. Though some of them are already tabulated, many data has been presented in various manner and not in the same criteria, some are based on detailed studies of numerous samples from rock units of well defined geological age whereas others are based on a preliminary studies and with unknown geological age. It is shown through the present tabulation how large is the diversity among presented data. The present collection is the first trial and, therefore, any ambiguous values in the table would be corrected with the aid of authors referred in the last column of each table.

\section{Archeomagnetic and Paleomagnetic Intensity (Table 1 page, 3-6)}

Geomagnetic intensity data of the past are not many, therefore, all the data are arranged in one table. Age of the specimen is expressed in year AD, whereas a small part of materials of geological past is clasified by geological period and its age is given by million years (my) before present (BP).

The table contains 7 columns.

Column 1 gives age or geological period of studied materials. Methods of age determination are noted in column 6 as will be mentioned below.

Column 2 gives the name and the kind of materials and the location of the specimens specified by district anc prefecture where possible. In many cases it is not possible to know exact location from the reference and only the name of the materials is noted.

Column 3 gives the number of specimens studied.

Column 4 gives the experimental value of $F / F_{0}$ where $F_{0}$ denotes the total force of the pre-

* Present address; Department of Geophysics and Atmospheric Sciences, University of Washington Seattle, Washington 98105, U.S.A. 
sent geomagnetic field and $F$ means the total force of the past geomagnetic field at the time and location of formation of (thermo-) natural remanent magnetization.

Column 5 gives the absolute intensity of the varitual geomagnetic dipole moment (CGS. $\mathrm{cm}^{3}$ ) at assigned age of the specimen. In many cases, however, the value is not shown according to the lack of original data in the references.

Column 6 gives the remarks where needed. Methods of age determination are noticed as follows; C14-carbon fourteen method. G-geological estimation. KA-potassium argon method. H-historical description.

Column 7 gives the number of references attatched on its end.

\section{Archeomagnetic Direction Data (Table 2, Page 7 15)}

Column 1 gives the age of used materials in AD. Negative value corresponds to the age of BC. In some cases where the age are shown as $x-y$, the definite age including any ambiguities is not given by referred authors and, hence, the present author has read them from the tables or figures in the references.

Column 2 gives the materials studied and locations of specimens specified by district and prefecture where possible.

Column $3(N)$ gives the number of specimens studied.

Column 4 (I) gives the inclination of the natural remanent magnetization of the specimens in units of degree where the positive value corresponds to the downward dipping vectors from the horizontal plane.

Column $5(D)$ gives the declinations in nuits of degree with positive sign for clockwise rotation from the geographic north.

Column $6\left(\alpha_{95}\right)$ gives the conical angle of the Fisher's 95\% confidence circle. (Ref. 18)

Column $7 \quad(k)$ gives the convergence factor of the unit vector of the natural remanent magnetization defined by Fisher.

Column 8 (VPP) gives the geographic position of the geomagnetic vertual dipole moment. Lat. is the geographic latitude expressed in units of degree with the positive value towards the north pole and the negative value towards the south pole. Long. is the geographic longitude with positive value for east-ward rotation.

Column 9 gives the remarks and the supplements to the magnetic data. NRM denotes that the magnetic data are only based upon natural remanent magnetization. Anatural remanent magnetization after ac field cleaning where -?- means that the peak ac field is not avairable at the present. *-magnetic data are reduced to the geomagnetic field at Tokyo. **-magnetic data are reduced to the geomagnetic field at $138^{\circ} \mathrm{E}$ and $37^{\circ} \mathrm{N}$ (observatory in Japan). C14-dated by carbon fourteen method. Data without $*, * *$ or C14 denote that the natural remanent magnetization are not corrected and dating was made by historical descriptions, types of associated potteries and/or geological key beds of known age. Column $\mathbf{1 0}$ gives the number of the reference in the same manner as column 7 of the intesity data. 


\section{Paleomagnetic Direction Data (Table 3, page 16 39)}

Data of paleomagnetic studies are avairable up to middle paleozoic period (Devonian). Magnetic data are arranged starting from recent rock units to the geoligical past. In the first place rocks of formations are classified in order of geological periods. The estimation of the age for rock units from geological view point shows wide varieties, for instance, some rock units are identified as of Tertiary period and others are as of upper Miocene time. Hence, rock units with larger ambiguity in its age are arranged in lower rows in the present table. The table contains 11 columns.

Column 1 gives ages of a rock units of year $\mathrm{AD}$ only for four cases with known age and in units of million years (BP) for all other cases. From column 2 to column 7 , the explanation is the same as for the archeomagnetic diretion data.

Column $8 \quad(P)$ gives the polarity of natural remanent magnetization. Obviously northward pointing and downward dipping unit vectors of the natural remanent magnetization are assigned as + , vectors with opposite direction are indicated by - and others with unidentifiable polarity are shown with $\mathrm{M}$.

Column 9 (VPP) corresponds to the column 8 in the archeomagnetic direction data.

Column 10 gives the remarks and supplements to the magnetic data. NRM indicates that the paleomagnetic data are obtained only from natural remanent magnetization. INTthe intensity data are avairable. Qn-the Königsberger's ratio. Ax-all the sample studied are demagnetized in the ac magnetic field with peak field $x(\mathrm{Oe})$. A-? - - peak acfield is not avairable TDx-thermal demagnetization for all the specimens studied up to $\mathrm{x}$ degrees in centigrade in a nonmagnetic space. No Mht.-specimens containing maghemite $\left(\gamma-\mathrm{Fe}_{2} \mathrm{O}_{3}\right.$ phase) are rejected. KA-age determinations were made through potassium argon method. ST-any kind of stability test is carried out for all the specimen. General trend removed-the standard regional magnetic anomaly is subtracted from the observed total force of the geomagnetic field obtained through survey.

Column 11 gives the number of referred papers in the same manner as before.

Table 1. Archeomagnetic and Paleomagnetic Intensity Data (page 3 6)

\begin{tabular}{|c|c|c|c|c|c|c|}
\hline Age (AD) & Site and Materials & $N$ & $F / F_{0}$ & $\operatorname{VDM}\left(\times 10^{25} \mathrm{CGS}\right)$ & Remarks & References \\
\hline 1962 & Basalt, Miyakejima & 5 & 1.02 & - & $\mathrm{H}$ & 45 \\
\hline 1951 & " , Ōshima, & 6 & 1.12 & - & $\mathrm{H}$ & $" 1$ \\
\hline 1783 & ", Asama Volcano & 6 & 1.15 & - & $\mathrm{H}$ & $" 1$ \\
\hline $1780 \pm 5$ & Andesite, Sakurajima & 1 & 1.11 & - & $\mathrm{H}$ & 78,81 \\
\hline 1778 & Basalt, Sakurajima & 5 & $1.20 \pm 0.06$ & - & $\mathrm{H}$ & 59 \\
\hline 1552 & $\prime \prime, \quad 11$ & 4 & $1.20 \pm 0.02$ & - & $\mathrm{H}$ & $" \prime$ \\
\hline 1281 & Basalt, Asama Volcano & 3 & 1.24 & - & $\mathrm{H}$ & 45 \\
\hline $1250 \pm 80$ & Baked earth, Toyota & 4 & $1.23 \pm 0.01$ & - & $\mathrm{H}$ & 78,81 \\
\hline $1470 \pm 5$ & Andesite, Sakurajima & 1 & 1.24 & - & $\mathrm{H}$ & $" 11$ \\
\hline 1421 & Basalt, Sakurajima & 6 & $1.33 \pm 0.08$ & - & $\mathrm{H}$ & 59 \\
\hline $1300 \pm 30$ & " , Sakurajima & 4 & $1.09 \pm 0.07$ & - & $\mathrm{H}$ & $\prime \prime$ \\
\hline $1120 \pm 20$ & Baked earth, Seto & 4 & $1.26 \pm 0.02$ & - & & 78,81 \\
\hline $1070 \pm 70$ & Basalt, Seto & 4 & $1.02 \pm 0.05$ & - & & 59 \\
\hline
\end{tabular}




\begin{tabular}{|c|c|c|c|c|c|c|c|c|}
\hline Age (AD) & \multicolumn{3}{|c|}{ Site and Materials } & $N$ & $F / F_{0}$ & $\operatorname{VDM}\left(\times 10^{25} \mathrm{CGS}\right)$ & Remarks & References \\
\hline $1000 \pm 20$ & \multicolumn{3}{|c|}{ Baked earth, Seto } & 4 & $1.45 \pm 0.03$ & - & & 78,81 \\
\hline $650 \pm 50$ & \multicolumn{3}{|c|}{ " , Sakai } & 2 & $1.48 \pm 0.01$ & - & & $11, " 1$ \\
\hline $950 \pm 50$ & \multicolumn{3}{|c|}{$" 1 "$} & 2 & $140+0.19$ & - & & $11, \quad 11$ \\
\hline $920 \pm 20$ & " & , Seto & & & & & & 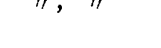 \\
\hline $765 \pm 15$ & \multicolumn{3}{|c|}{ Tile, Seto } & 4 & $1.08 \pm 0.03$ & - & & 59 \\
\hline $735 \pm 15$ & \multicolumn{3}{|c|}{ Tile, Seto } & 3 & $1.05 \pm 0.03$ & - & & $"$ \\
\hline $725 \pm 25$ & \multicolumn{3}{|c|}{ Tile, Sakai } & 5 & $1.43 \pm 0.04$ & - & & 78,81 \\
\hline $710 \pm 60$ & \multicolumn{3}{|c|}{ Baked earth, Sakai } & 3 & $1.21 \pm 0.06$ & - & & $11, " 1$ \\
\hline $565 \pm 15$ & \multicolumn{3}{|c|}{ Pottery, Sakai } & 4 & $1.14 \pm 0.03$ & - & & 59 \\
\hline $550 \pm 20$ & \multirow{2}{*}{\multicolumn{3}{|c|}{ Baked earth, Sakai }} & 5 & $146+003$ & - & & 78.81 \\
\hline $540 \pm 40$ & & & & 5 & $1.46 \pm 0.03$ & - & & 18,81 \\
\hline $525 \pm 10$ & \multicolumn{3}{|c|}{ Pottery, Sakai } & 8 & $1.37 \pm 0.03$ & - & & 59 \\
\hline $480 \pm 20$ & \multicolumn{3}{|c|}{ Baked earth, Tsu } & 3 & $1.44 \pm 0.06$ & - & & 78,81 \\
\hline $380 \pm 20$ & \multicolumn{3}{|c|}{ Clay Idol, Ueno } & 2 & $1.35 \pm 0.09$ & - & & $" 1$, \\
\hline $300 \pm 100$ & \multicolumn{3}{|c|}{ Basalt, Ueno } & 5 & $1.53 \pm 0.13$ & - & & 59 \\
\hline $220 \pm 20$ & \multicolumn{3}{|c|}{ Pottery, Hiraoka } & 2 & $1.41 \pm 0.01$ & - & & 78,81 \\
\hline $100 \pm 50$ & \multicolumn{3}{|c|}{ " , Fukuoka } & 1 & 1.43 & - & & $11, " 1$ \\
\hline$-100 \pm 50$ & \multicolumn{3}{|c|}{ " , Daitō } & 1 & 1.64 & - & & $\|, " 1$ \\
\hline$-150 \pm 150$ & \multicolumn{3}{|c|}{ Basalt, Daitō } & 2 & $1.54 \pm 0.01$ & - & & 60 \\
\hline$-300 \pm 250$ & \multicolumn{3}{|c|}{$\prime \prime, \quad " 1$} & 4 & $1.22 \pm 0.07$ & - & & $" 1$ \\
\hline-530 & \multicolumn{3}{|c|}{ Pottery, Araumi, East Japan } & 1 & 1.45 & 一 & & 36 \\
\hline " & " , & , 11 & $" 1$ & 1 & 1.16 & - & & $" \prime$ \\
\hline$-900 \pm 100$ & \multicolumn{3}{|c|}{ Pottery, Shiga } & 2 & $0.93 \pm 0.01$ & - & & 78,81 \\
\hline$-1000 \pm 400$ & \multicolumn{3}{|c|}{ Basalt, Shiga } & 1 & 1.00 & - & & 59 \\
\hline-1030 & Pottery, & Furuhara, E & st Japan & 1 & 0.88 & - & & 36 \\
\hline "1 & $" \prime$ & , , & $" \prime$ & 1 & 1.01 & - & & " \\
\hline$" \prime$ & $" 1$ & , 11 & $\prime \prime$ & 1 & 1.19 & - & & $" 1$ \\
\hline$" 1$ & " , & Takeda, & $\prime \prime$ & 1 & 0.42 & - & & " \\
\hline " & "I & , II, & $" \prime$ & 1 & 0.68 & - & & " \\
\hline " & $" 1$ & , II, & " & 1 & 0.82 & - & & " \\
\hline " & $" 1$ & , II, & $" \prime$ & 1 & 0.74 & - & & " \\
\hline$" 1$ & Pottery & Suginodō, E & st Japan & 1 & 0.86 & - & & " \\
\hline "1 & $" 1$ & , " , & $" 1$ & 1 & 1.01 & - & & " \\
\hline " & " & " & "I & 1 & 1.54 & - & & " \\
\hline$" 1$ & $"$ & " & $" \prime$ & 1 & 1.40 & - & & "1 \\
\hline "1 & " & , $\quad 11$ & " & 1 & 0.68 & - & & " \\
\hline "1 & " & $" 1$ & $" 1$ & 1 & 1.49 & - & & " \\
\hline$-2000 \pm 500$ & Basalt, & "l , & " & 1 & 0.94 & - & $\mathrm{C} 14$ & $"$ \\
\hline-2000 & " , & Asama Volce & & 3 & 1.83 & - & " & "1 \\
\hline-2330 & Pottery & , Mukōdai, E & st Japan & 1 & 0.56 & - & "1 & " \\
\hline " & " & " , & $" \prime$ & 1 & 0.70 & - & $" 1$ & $" 1$ \\
\hline$" 1$ & $"$ & , " & $" 1$ & 1 & 0.67 & - & "l & " \\
\hline-2470 & $"$ & Atamadai, & " & 1 & 0.64 & - & $" 1$ & " \\
\hline "I & "1 & $" 1$ & "I & 1 & 0.65 & - & " & "1 \\
\hline$" 1$ & $" 1$ & $" \prime$ & $" 1$ & 1 & 0.66 & - & $" \prime$ & $" \prime$ \\
\hline
\end{tabular}




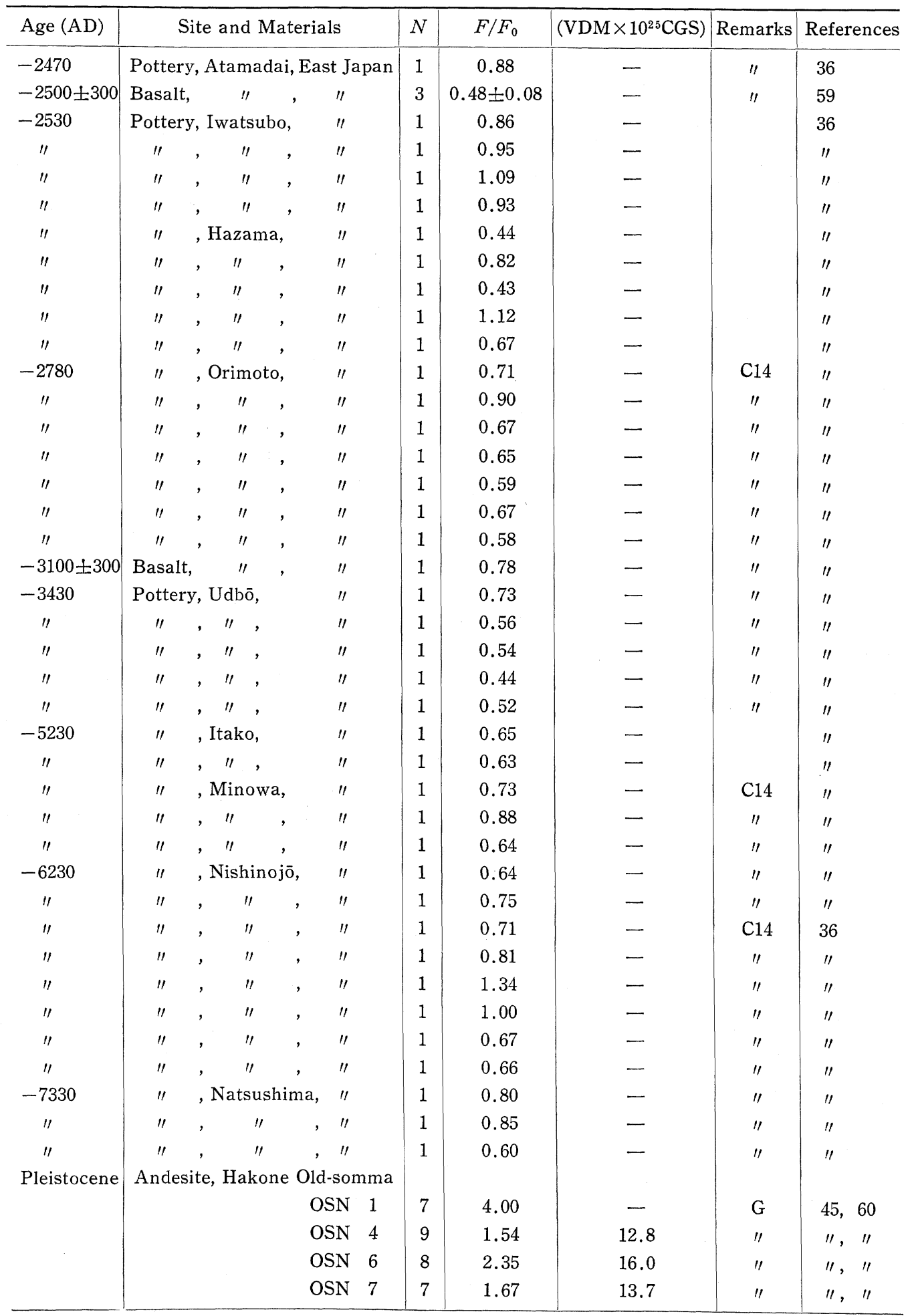




\begin{tabular}{|c|c|c|c|c|c|c|}
\hline Age (my.) & Site and Materials & $N$ & $F / F_{0}$ & $\operatorname{VDM}\left(\times 10^{25} \mathrm{CGS}\right)$ & Remarks & References \\
\hline & OSN 8 & 8 & 2.20 & 18.7 & " & $" 1 "$ \\
\hline & OSN 17 & 7 & 1.91 & 15.6 & $" 1$ & $\|, "\|$ \\
\hline & OSN 18 & 7 & 1.73 & 14.4 & $" \prime$ & $" 11$ \\
\hline & OSN 24 & 7 & 0.30 & 11.5 & " & $", "$ \\
\hline \multirow[t]{6}{*}{ Pleistocene } & Kujū Volcanic Rocks, Kyushu & & & & & \\
\hline & $\mathrm{KP}$ & 2 & - & 5.0 & $" \prime$ & 82,45 \\
\hline & $\mathrm{D} 4$ & 4 & - & 10.1 & $" \prime$ & $" 1 "$ \\
\hline & K7 & 5 & - & 9.9 & $" \prime$ & $", " 1$ \\
\hline & $\mathrm{K} 4$ & 3 & - & 11.9 & $" \prime$ & $" 1 "$ \\
\hline & $\mathrm{Dh}$ & 4 & - & 9.5 & $" \prime$ & $" 1$, \\
\hline \multirow[t]{3}{*}{ Pleistocene } & Usami Basalt, Izu & & & & & \\
\hline & UV06 & 5 & 0.82 & 5.5 & $(G)$ & 42,45 \\
\hline & UV07 & 7 & 1.56 & 13.5 & $\prime \prime$ & $" 1 "$ \\
\hline \multirow[t]{2}{*}{0.71} & UV08 & 5 & 1.44 & 12.3 & KA & $" 11,22$ \\
\hline & UV09 & 6 & 1.00 & 9.5 & (G) & $n, \quad "$ \\
\hline \multirow[t]{3}{*}{$0.45-0.66$} & UV10 & 8 & 1.80 & 17.4 & KA & $" 1 ", 22$ \\
\hline & UV11 & 5 & 1.10 & 8.5 & (G) & $11, " 1$ \\
\hline & UV17 & 5 & 1.80 & 16.8 & $" \prime$ & $" 1 "$ \\
\hline \multirow[t]{4}{*}{0.82} & UV12 & 9 & 1.02 & 9.3 & $\mathrm{KA}$ & $\|, \quad\|, 22$ \\
\hline & UV13 & 9 & 1.17 & 10.7 & (G) & $" 1 "$ \\
\hline & UV16 & 7 & 0.29 & 3.0 & $" \prime$ & $", " \prime$ \\
\hline & UV14 & 9 & 0.79 & 7.2 & " & $" 11,22$ \\
\hline \multirow[t]{7}{*}{ Pleistocene } & Dacite, Minakami-yama & 7 & 1.29 & - & G & 34 \\
\hline & Andesite, Hotaka, Gumma & 2 & - & 2.5 & " & 45 \\
\hline & Kitamatsuura Basalt, Kyushu & & & & & \\
\hline & KM21 & 2 & $0.18,0.08$ & mean1.5 & $\mathrm{G}(\mathrm{KA})$ & 45,72 \\
\hline & KM23 & 5 & 0.78 & 8.3 & " & $", " 1$ \\
\hline & KM52 & 4 & - & 8.8 & $" \prime$ & $", 74,72$ \\
\hline & KM52 & 5 & - & 10.6 & " & $" 1 ", "$ \\
\hline \multirow[t]{6}{*}{ Pliocene } & Kujū Volcanic Rocks, Kyushu & & & & & \\
\hline & $\mathrm{H} 1$ & 1 & - & 6.3 & G & 83,45 \\
\hline & $\mathrm{Rw}$ & 3 & - & 6.2 & $" \prime$ & $" 11$ \\
\hline & R1 & 1 & - & 8.7 & " & $" 11$ \\
\hline & $\mathrm{P} 1$ & 6 & - & 8.7 & " & $", " 1$ \\
\hline & $\mathrm{Tp}$ & 3 & - & 8.4 & " & $" 1$, \\
\hline \multirow[t]{9}{*}{ Neogene } & $\begin{array}{l}\text { Basaltic Andesite, Shibayama, } \\
\text { SF1 }\end{array}$ & 1 & 1.3 & - & $\prime \prime$ & 1 \\
\hline & $\mathrm{SF} 1$ & 1 & 0.4 & - & $" 1$ & $" 1$ \\
\hline & Altered Basalt, Shibayama & & & & & \\
\hline & SR1 & 1 & 1.5 & - & $" \prime$ & $" \prime$ \\
\hline & SR2 & 1 & 1.5 & - & $" \prime$ & "l \\
\hline & Andesite, Yashima, YR1 & 1 & 0.75 & - & "I & " \\
\hline & YR2 & 1 & 2.8 & - & $"$ & " \\
\hline & YR2 & 1 & 0.8 & - & " & "I \\
\hline & $\begin{array}{c}\text { Altered Andesite Shiroyama } \\
\text { KR1 }\end{array}$ & 1 & 0.8 & - & " & $" \prime$ \\
\hline
\end{tabular}




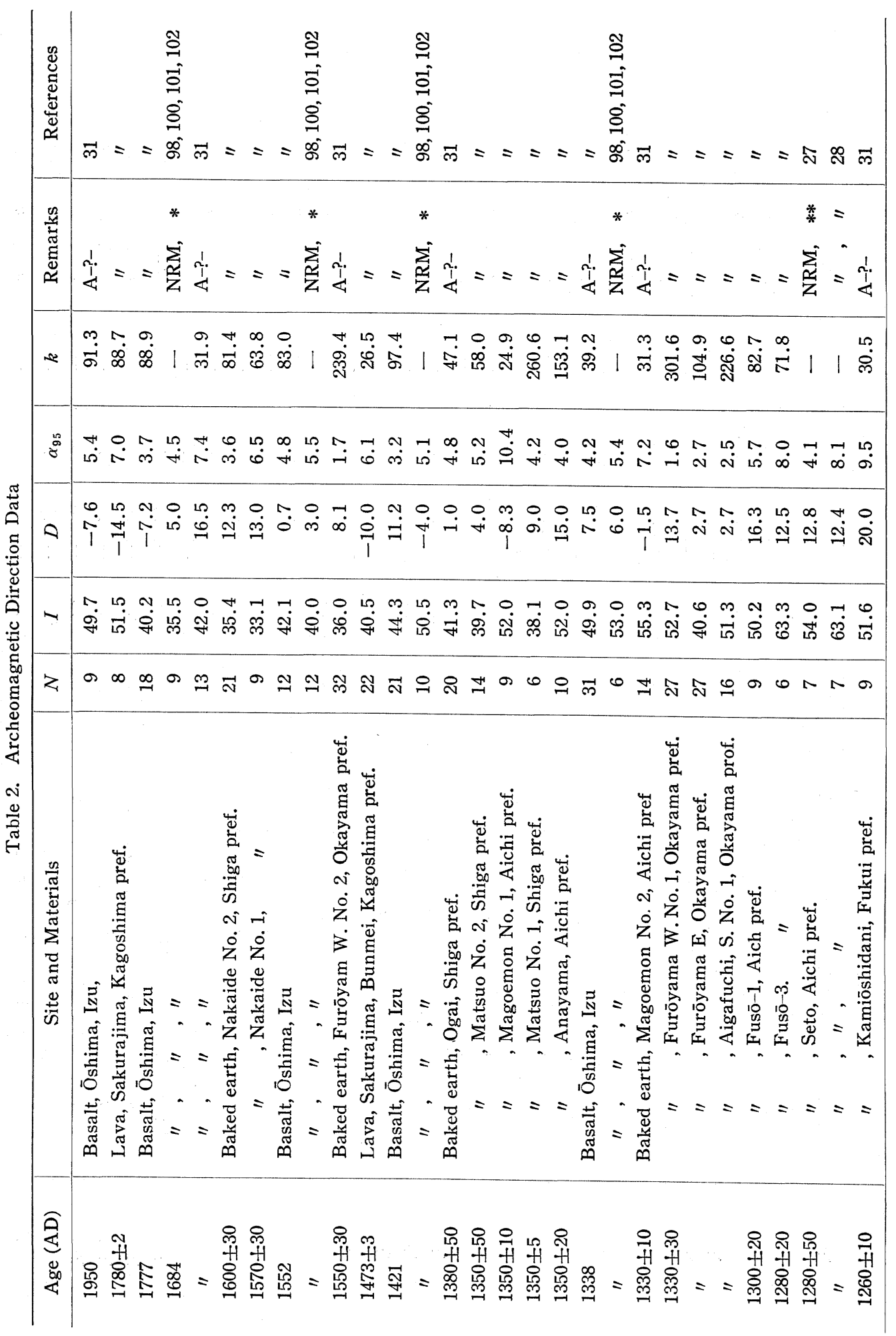




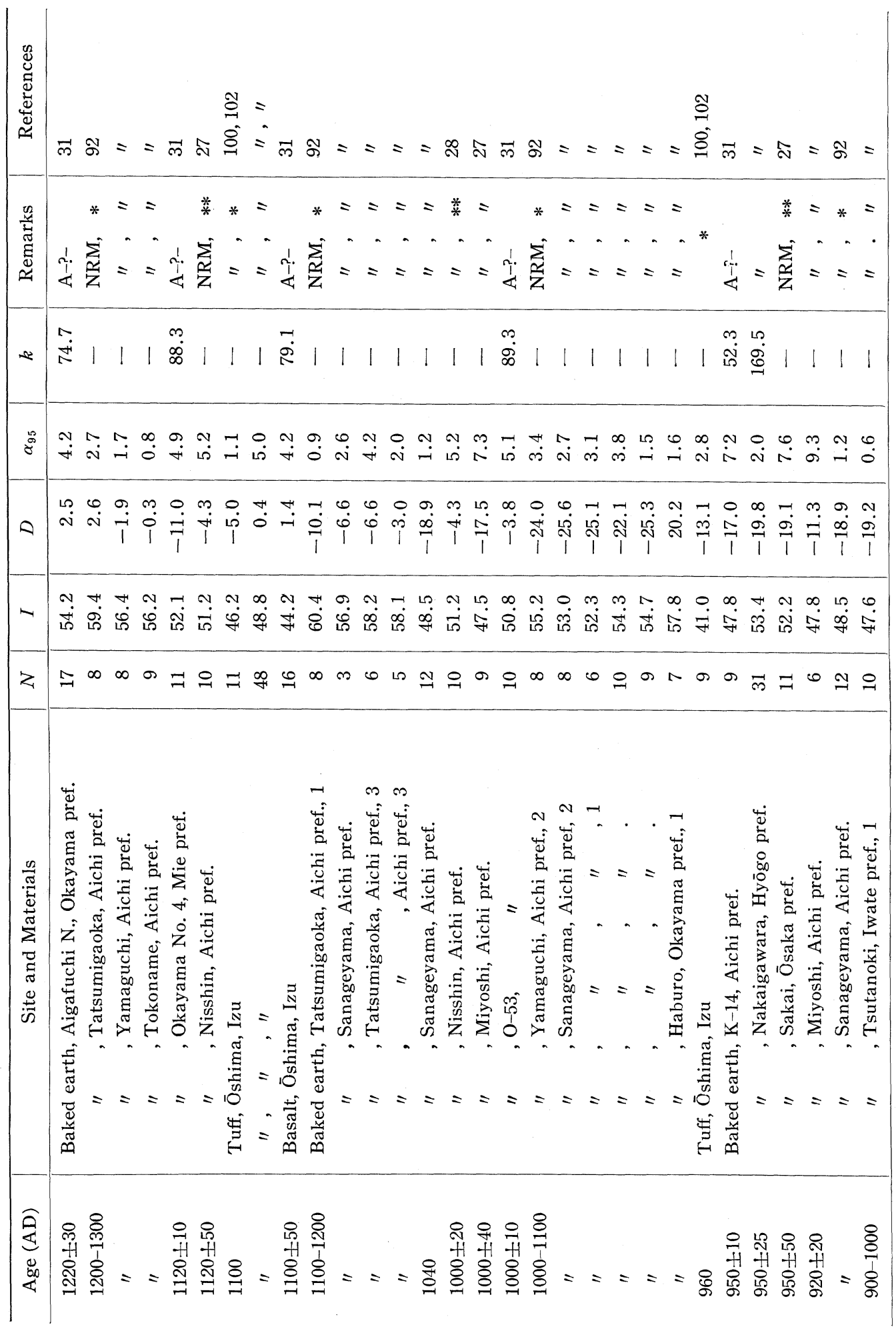




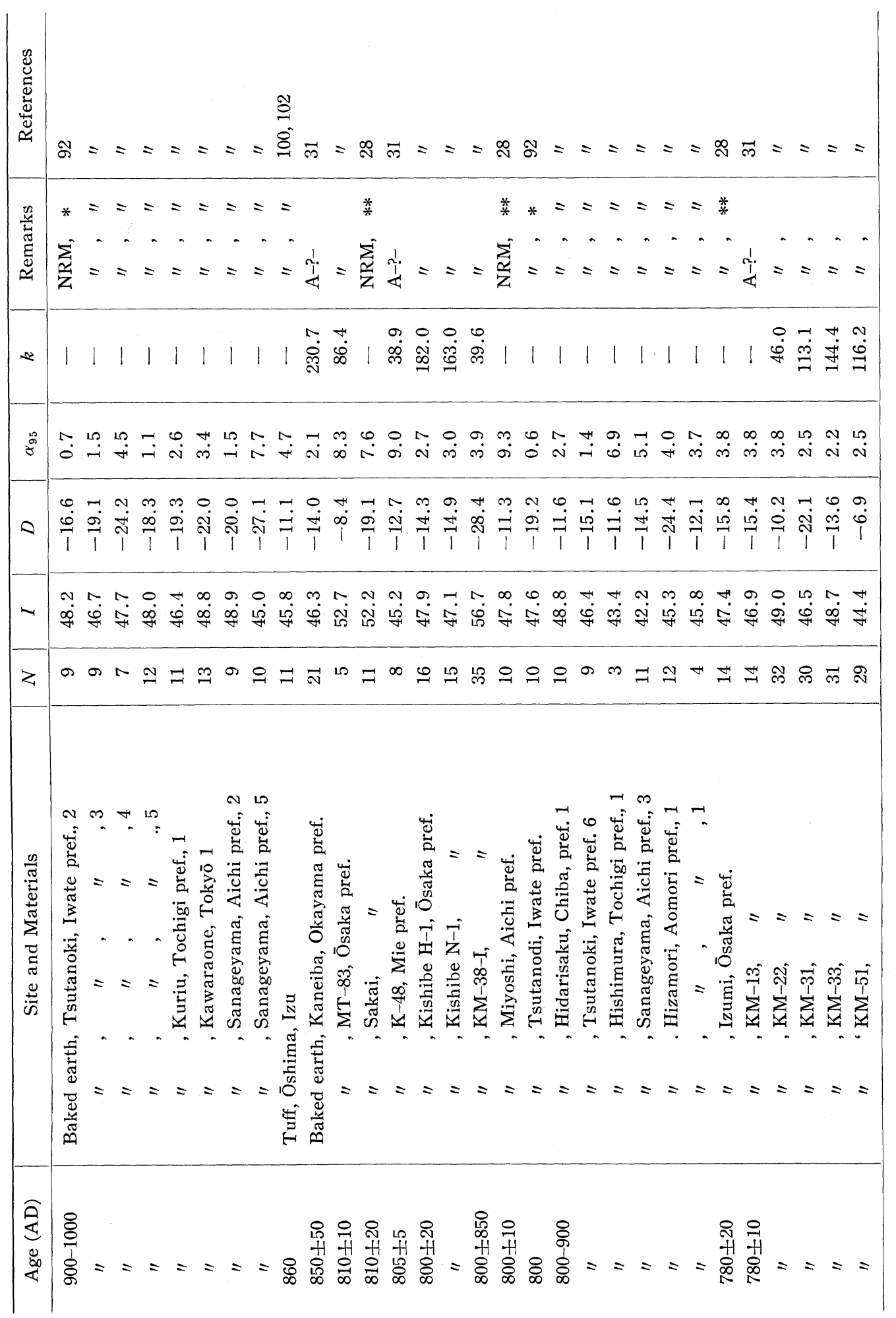




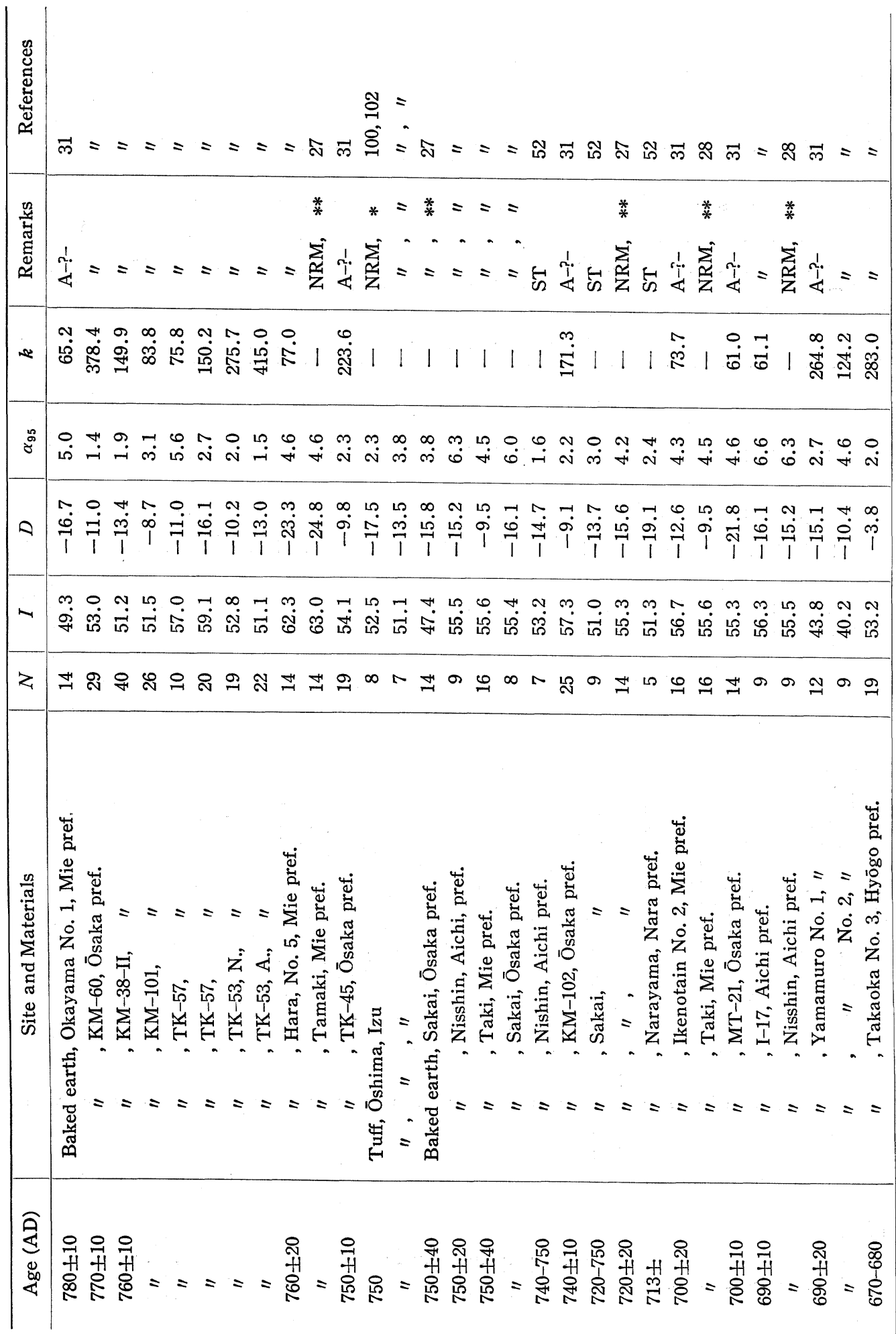




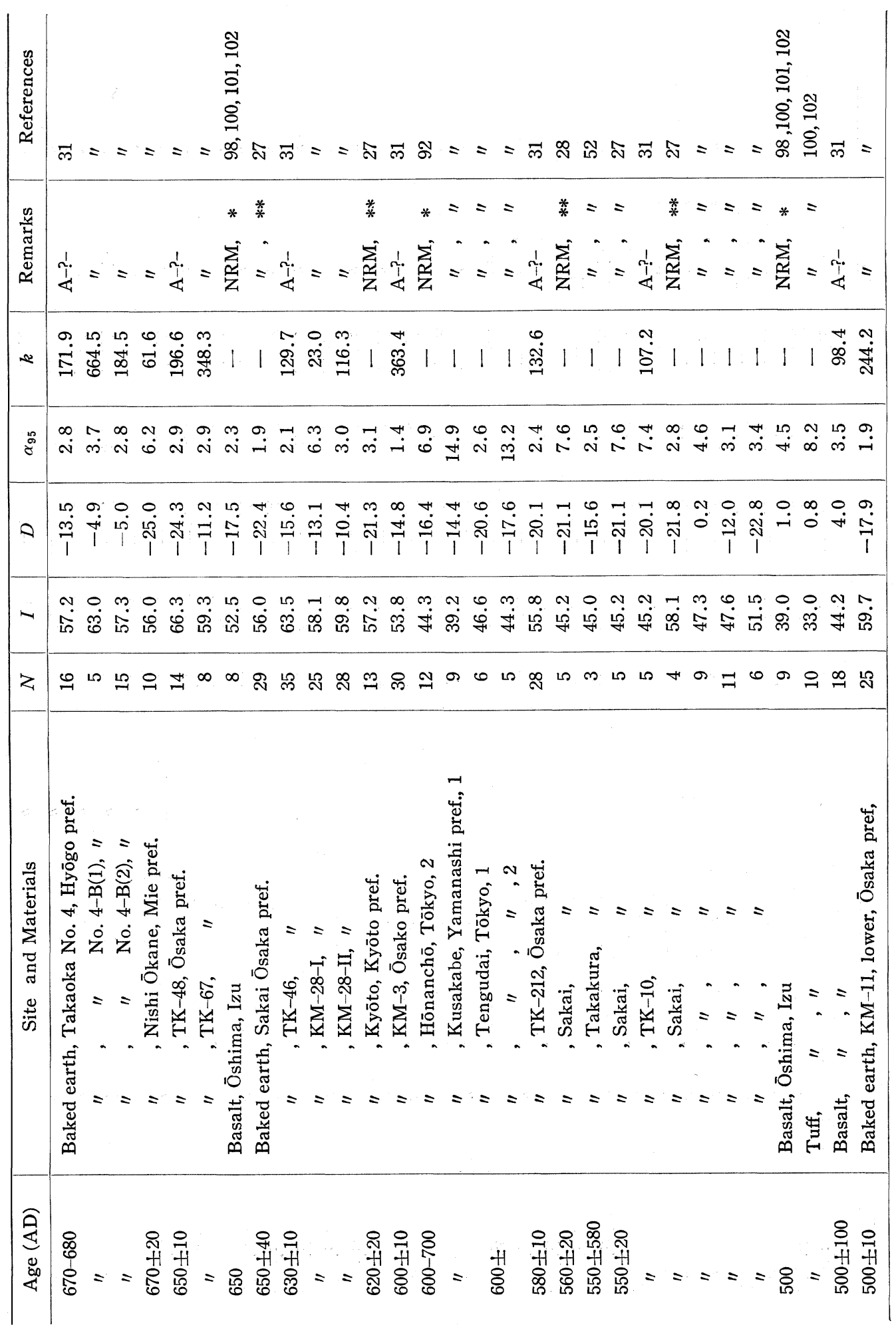




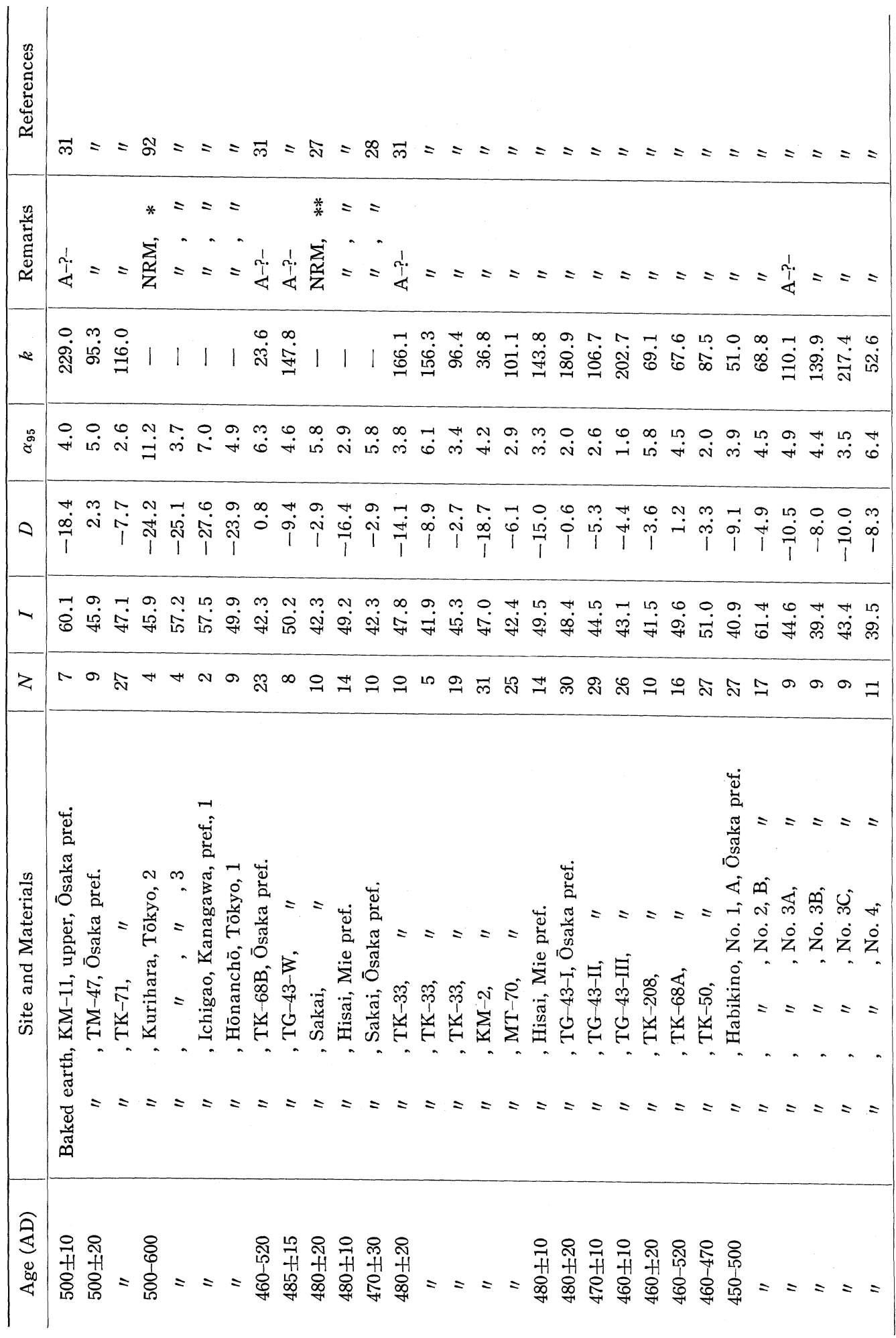




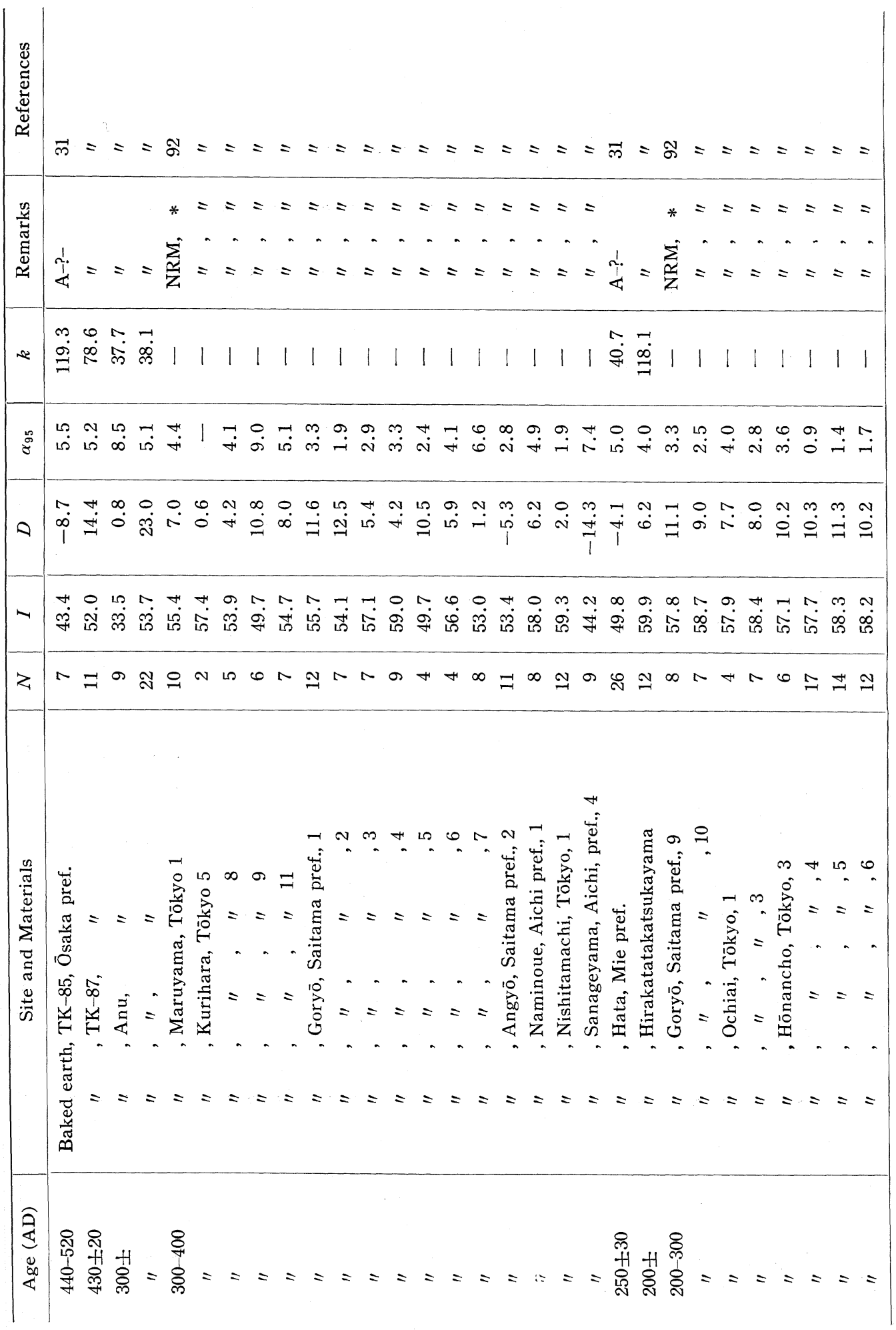




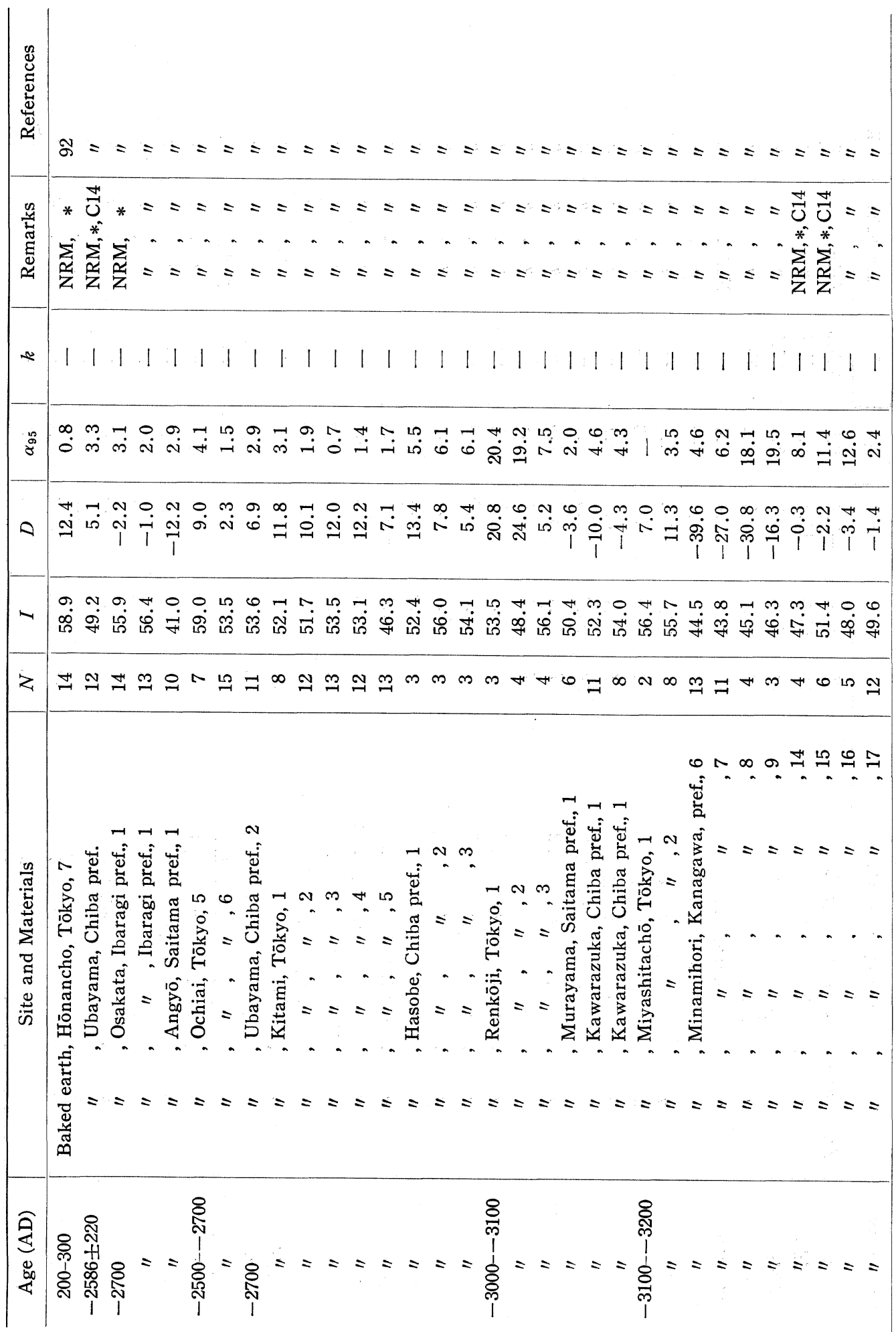




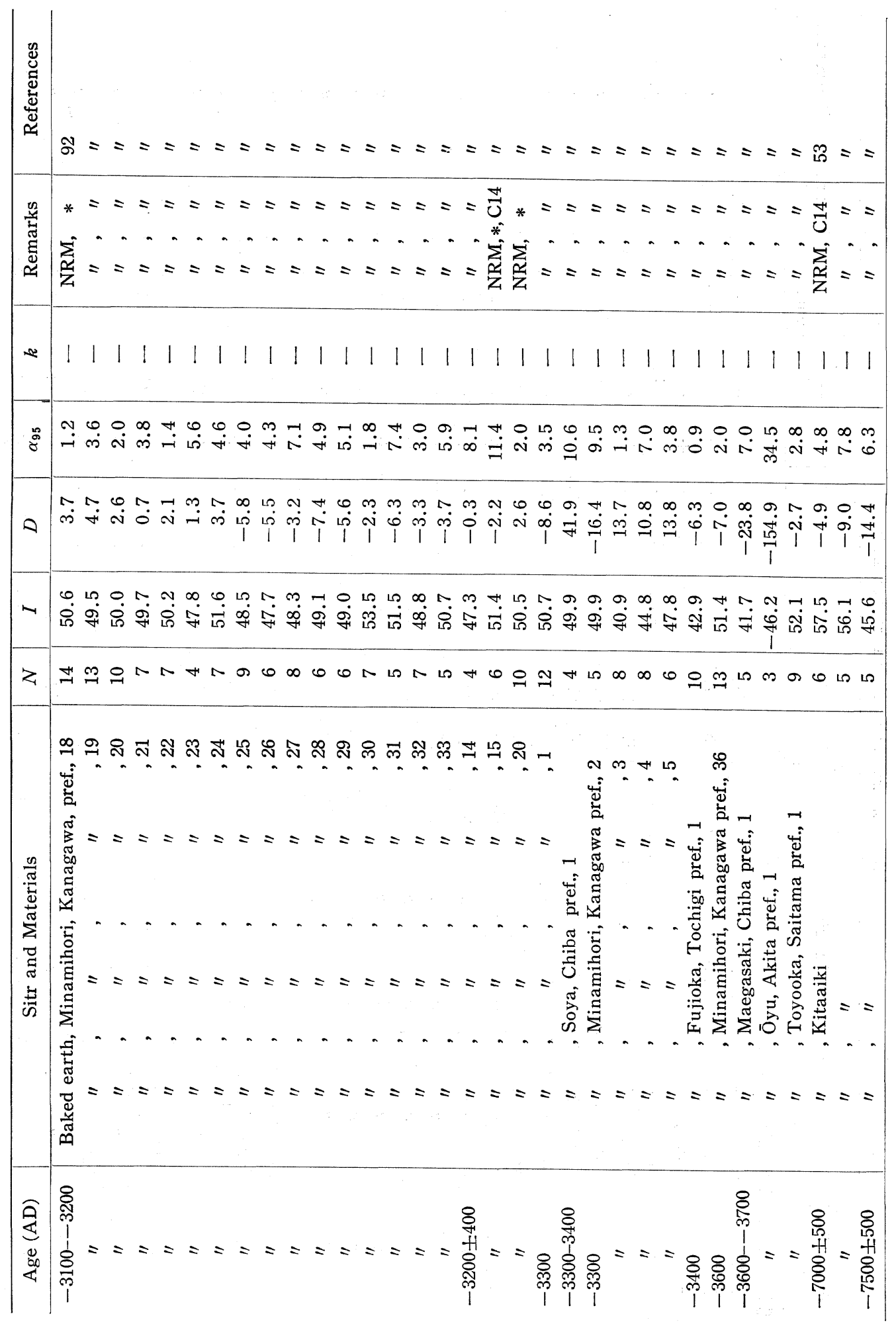




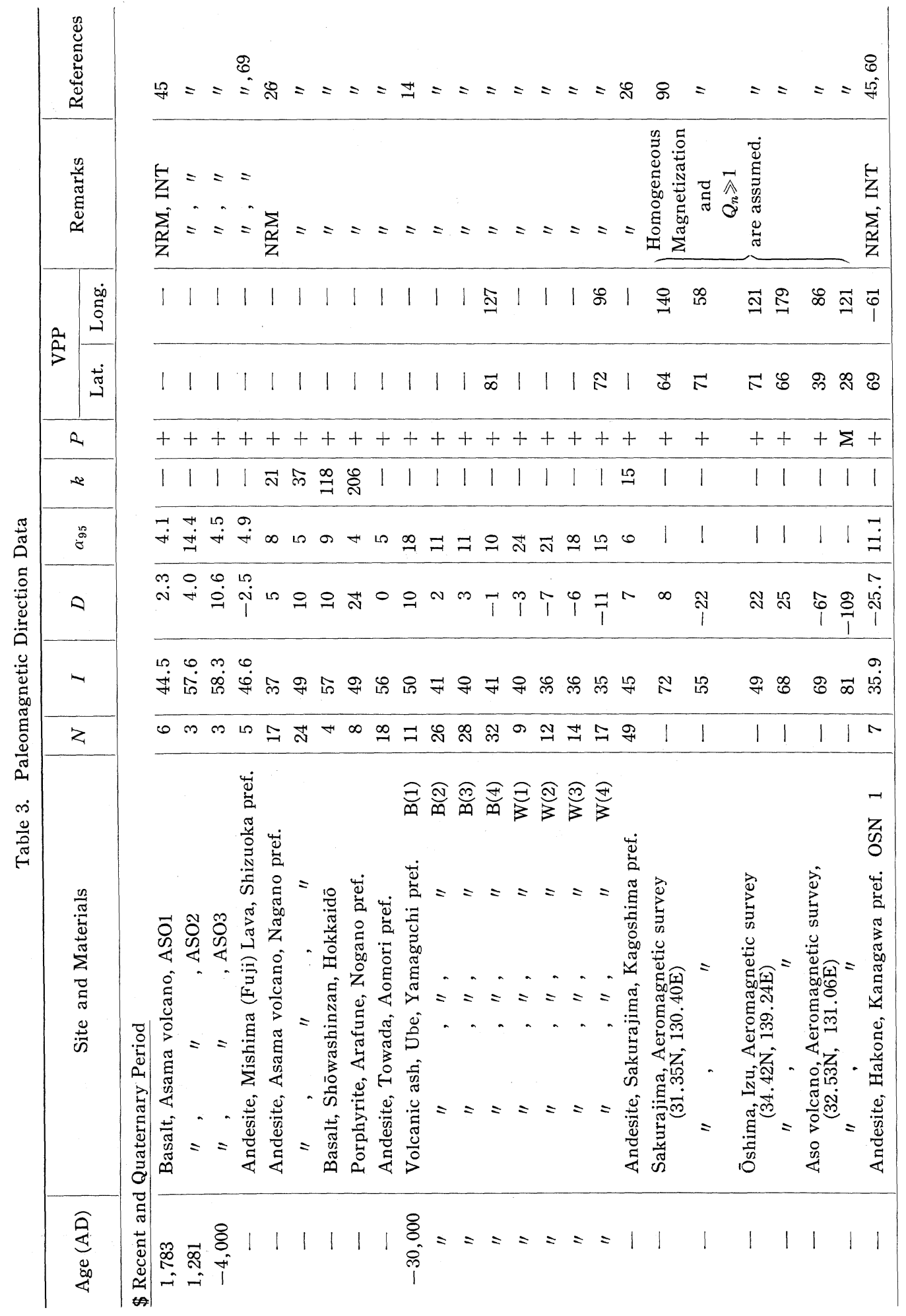




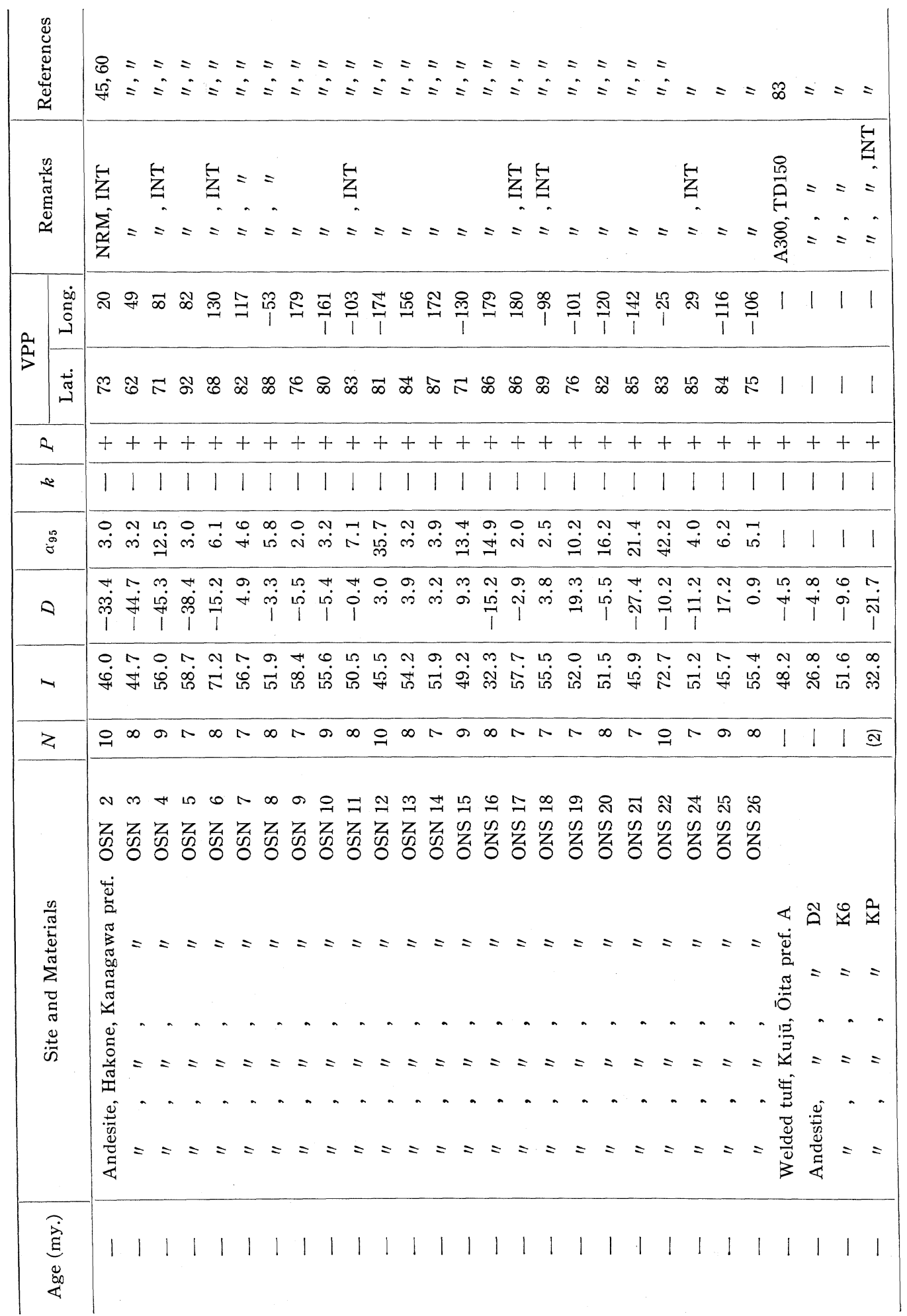




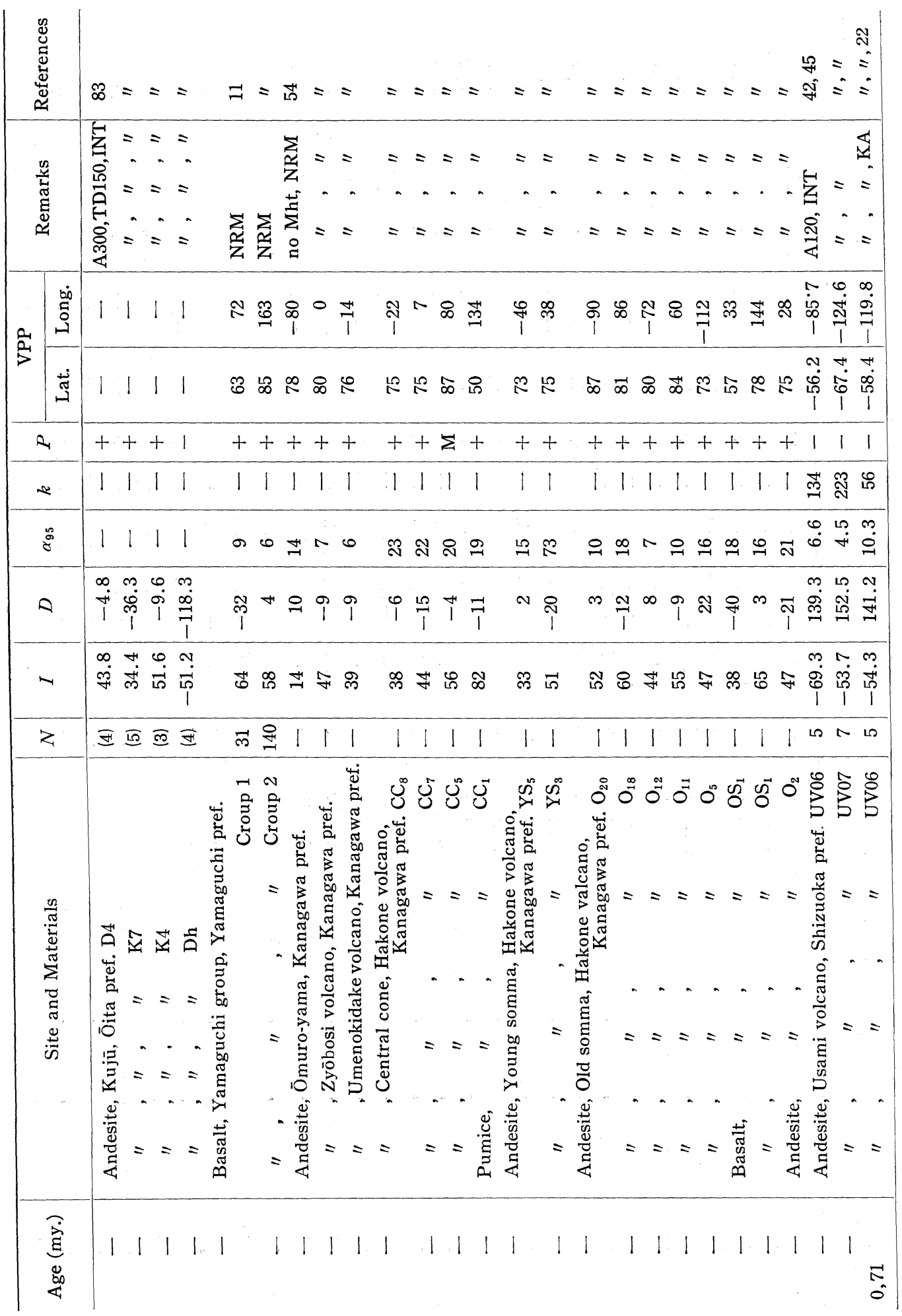




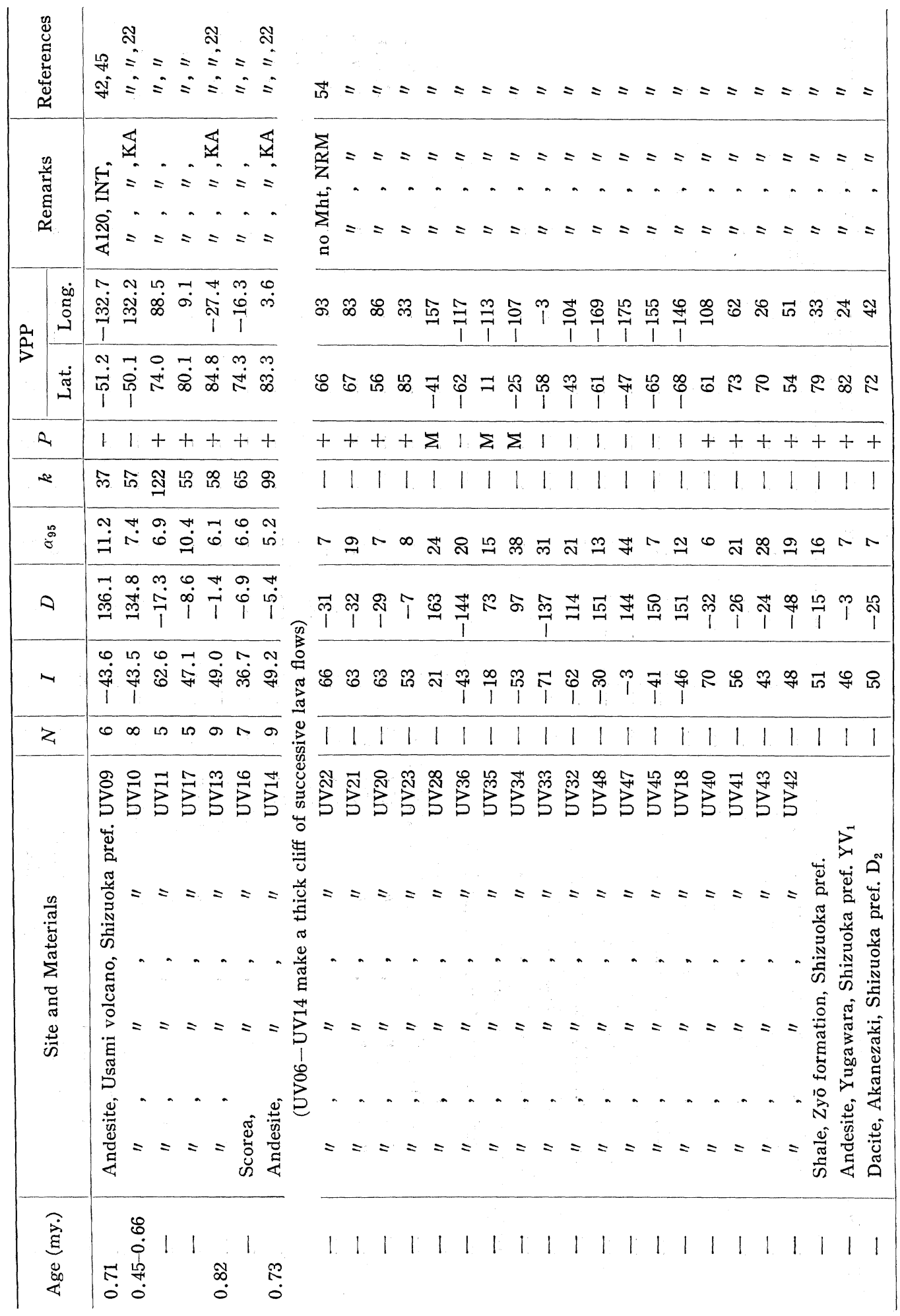




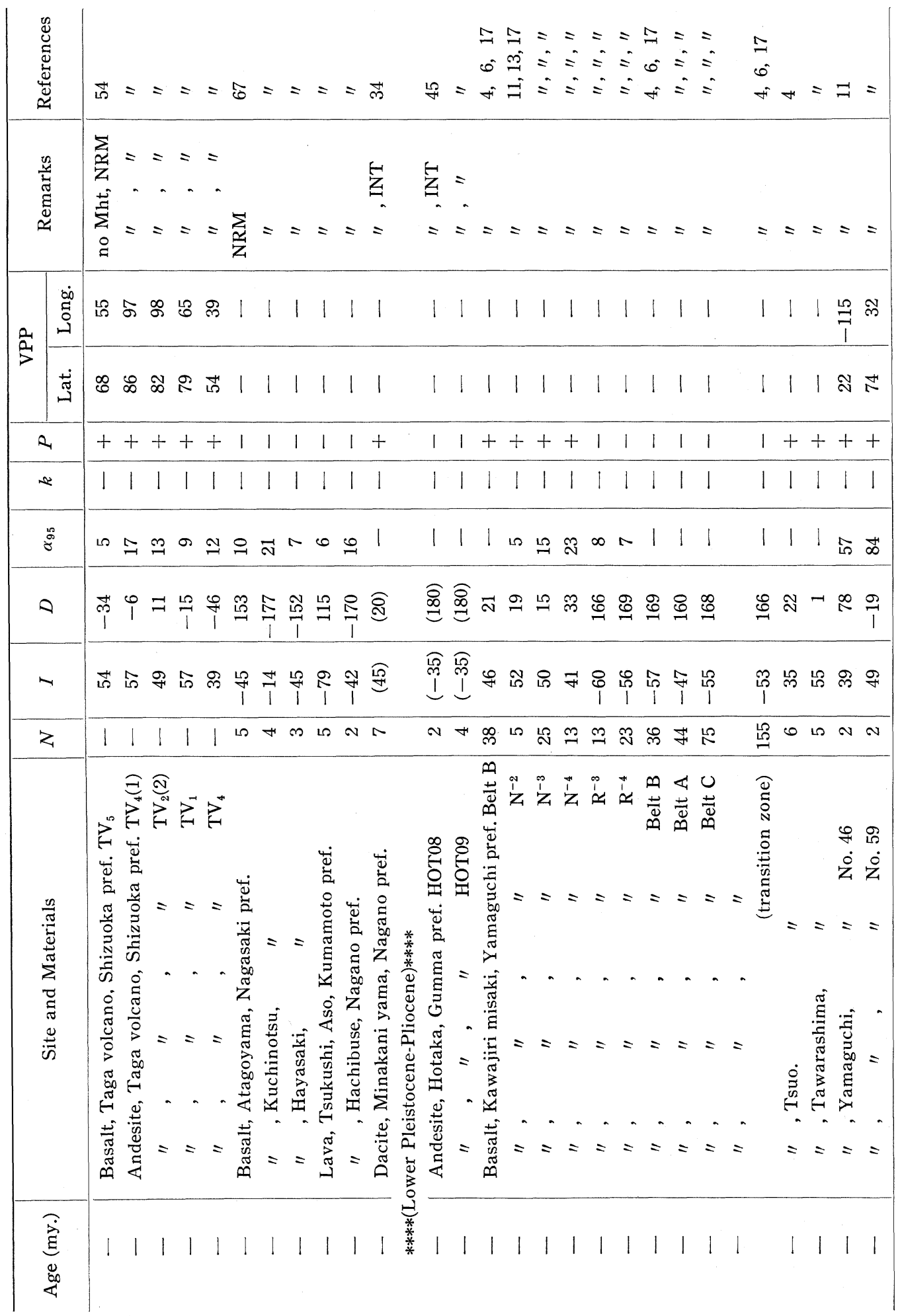




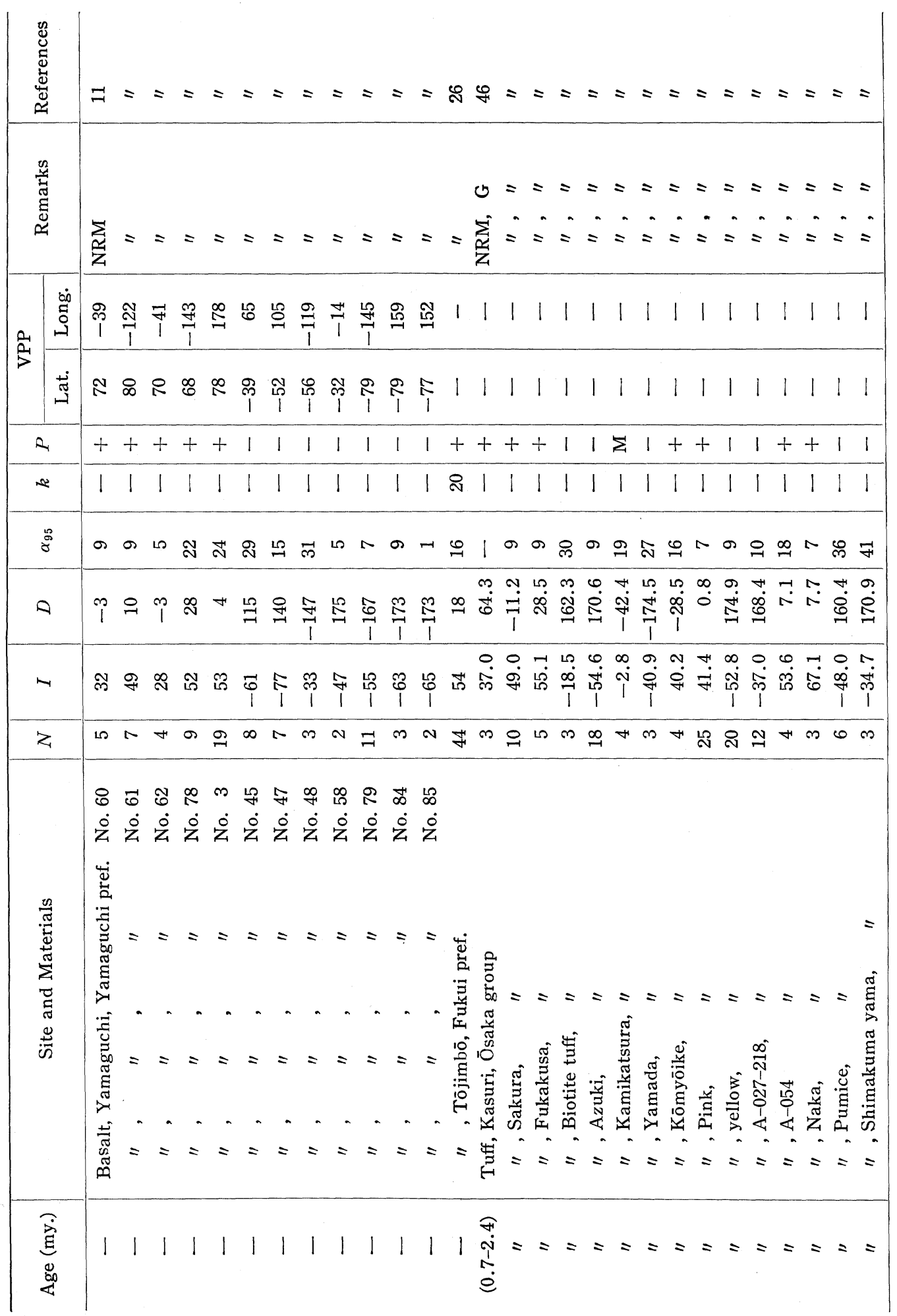




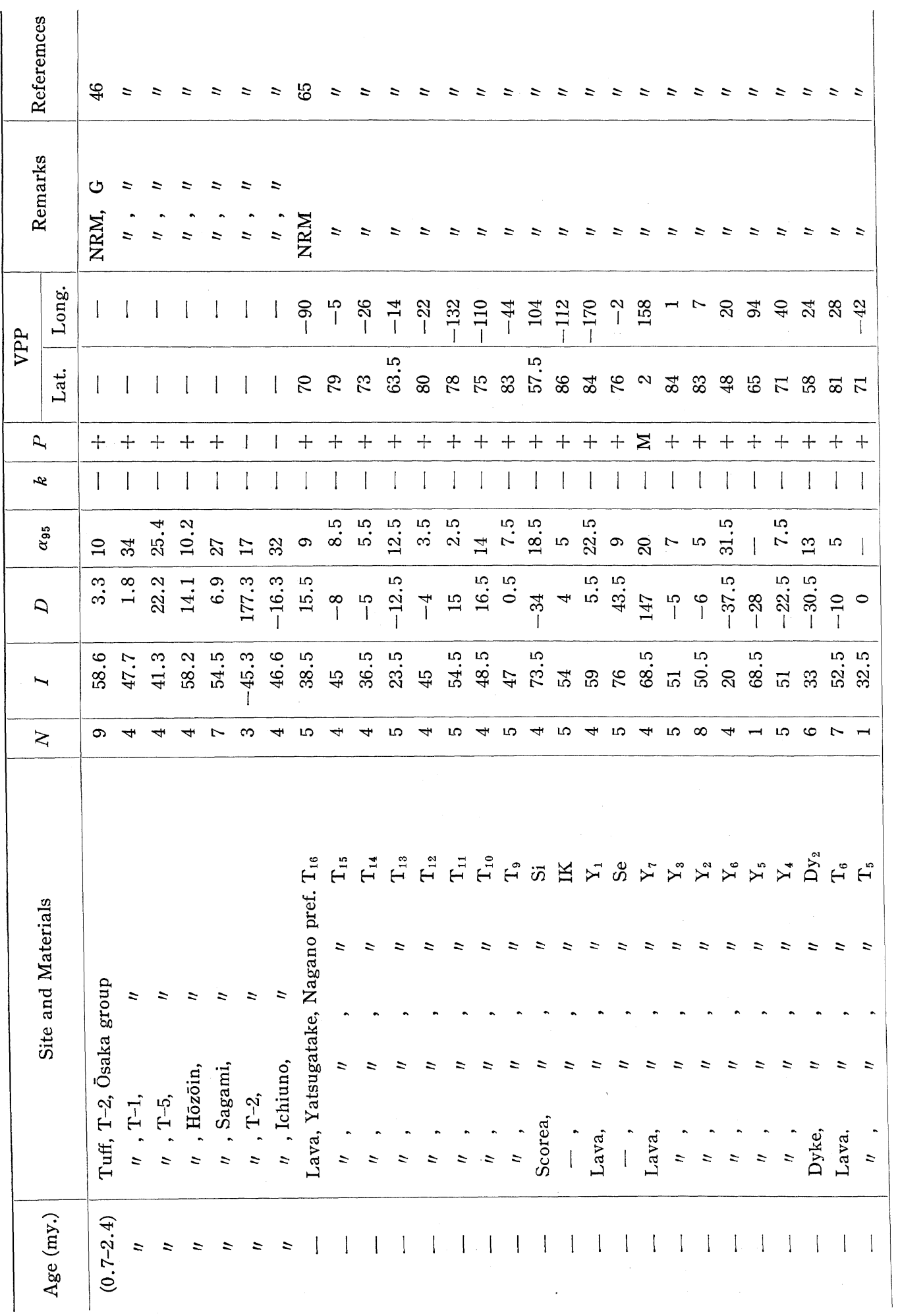




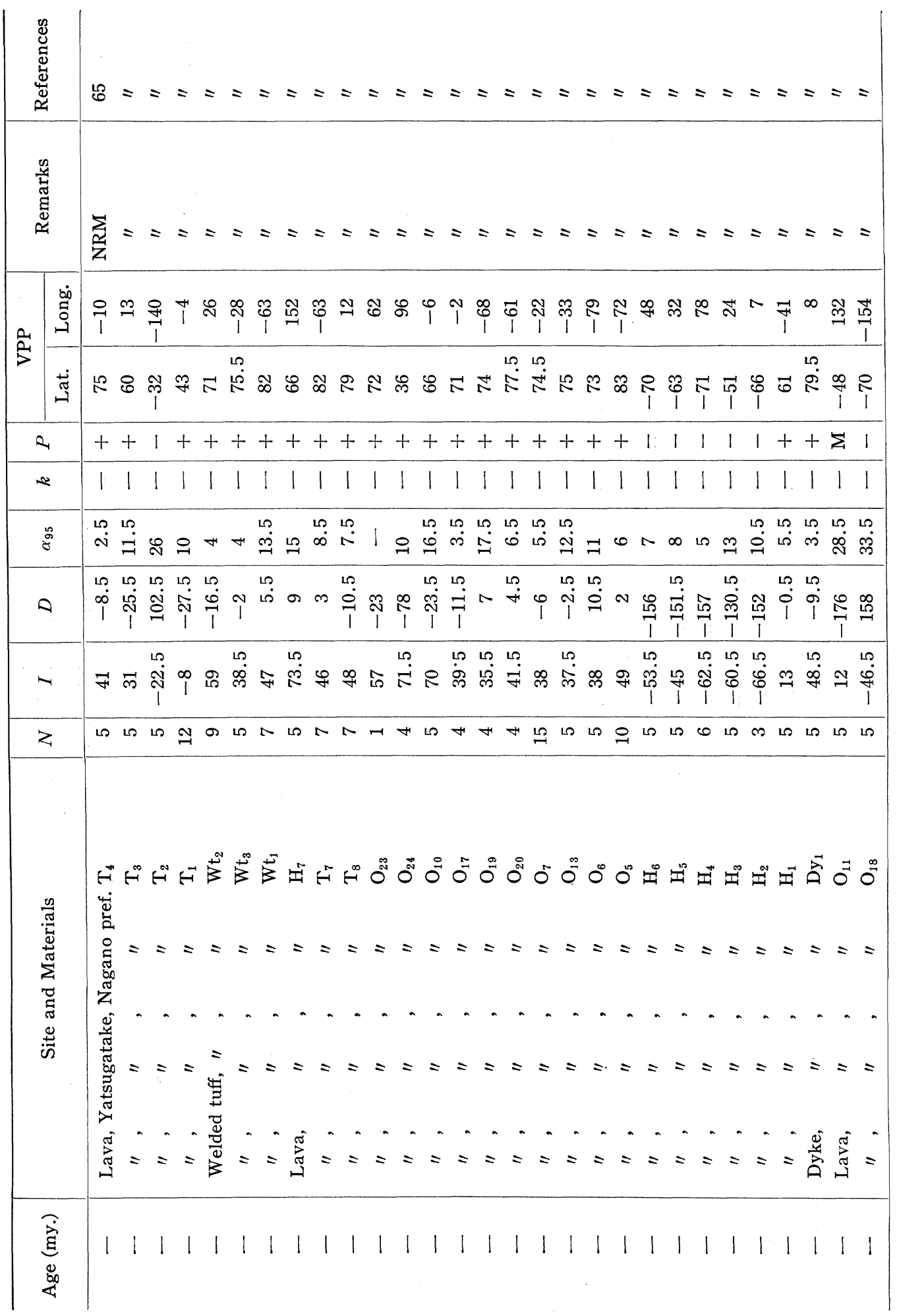




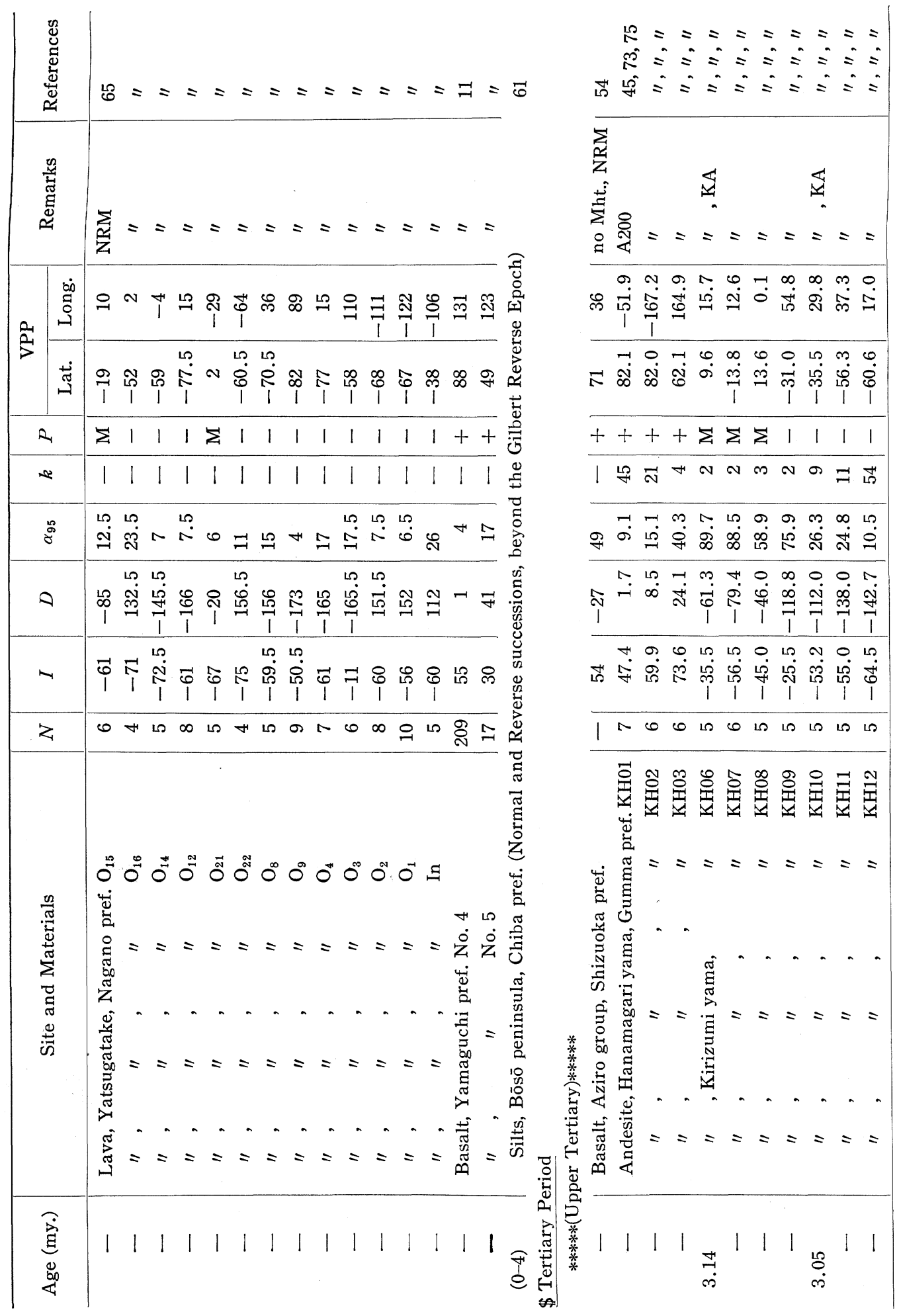




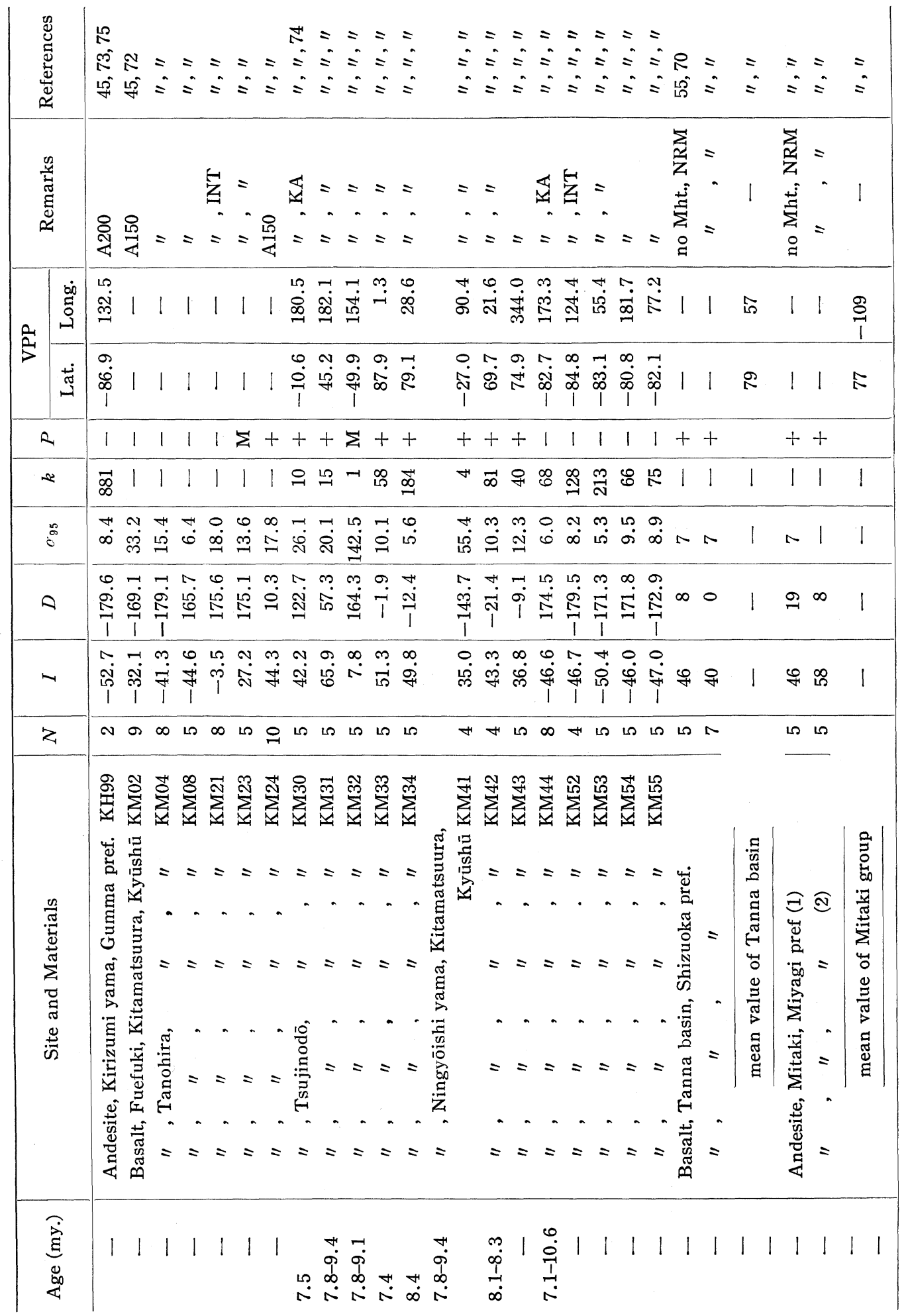




\begin{tabular}{|c|c|c|}
\hline 选 & 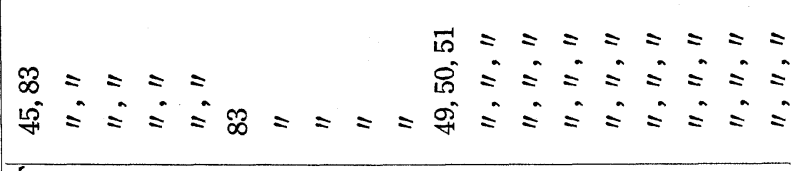 & 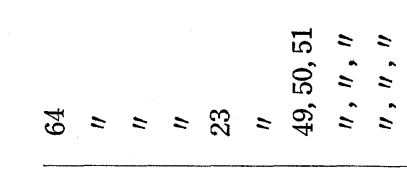 \\
\hline 离 & 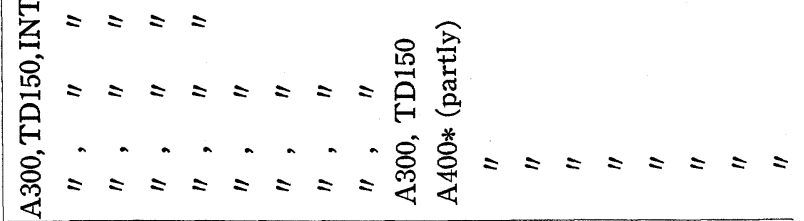 & 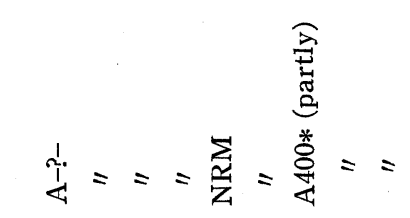 \\
\hline $\mid \begin{array}{l}\infty \\
\vdots \\
0 \\
\end{array}$ & 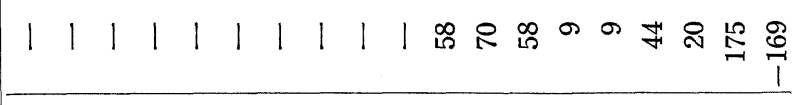 & 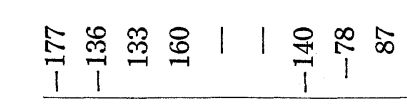 \\
\hline 嵌 & 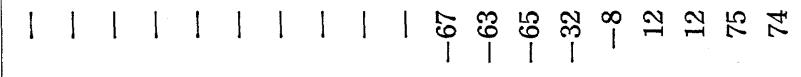 & 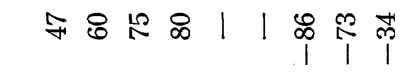 \\
\hline Q & $+11111++++11111 \sum \sum++$ & $++\Sigma \Sigma+111 \Sigma$ \\
\hline Ne & 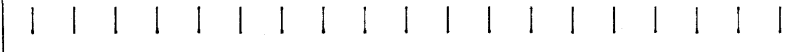 & $\begin{array}{lllllllll}1 & 1 & 1 & 1 & 1 & 1 & 1 & 1 & 1\end{array}$ \\
\hline$\stackrel{\mathscr{S}}{8}$ & 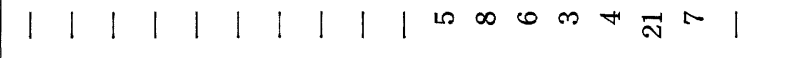 & $\cong$ L $10 \cong 0$ \\
\hline$\theta$ & 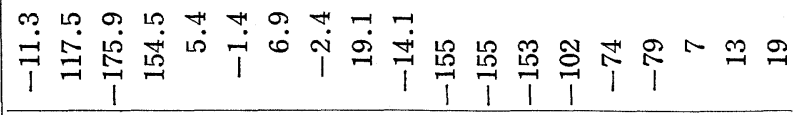 & 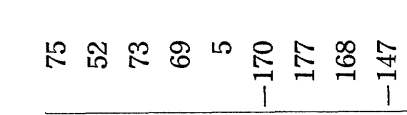 \\
\hline- & 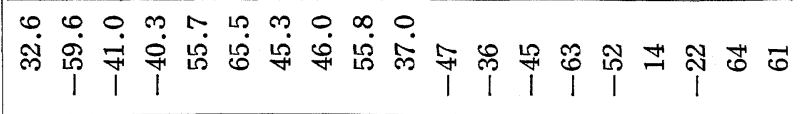 & $\infty$ 命 \\
\hline Z & 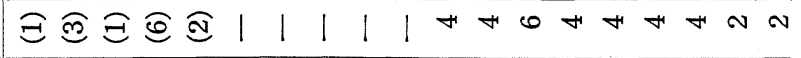 & 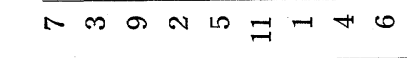 \\
\hline 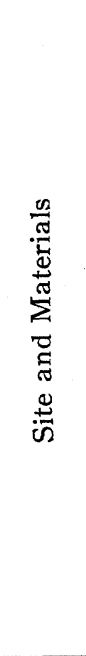 & 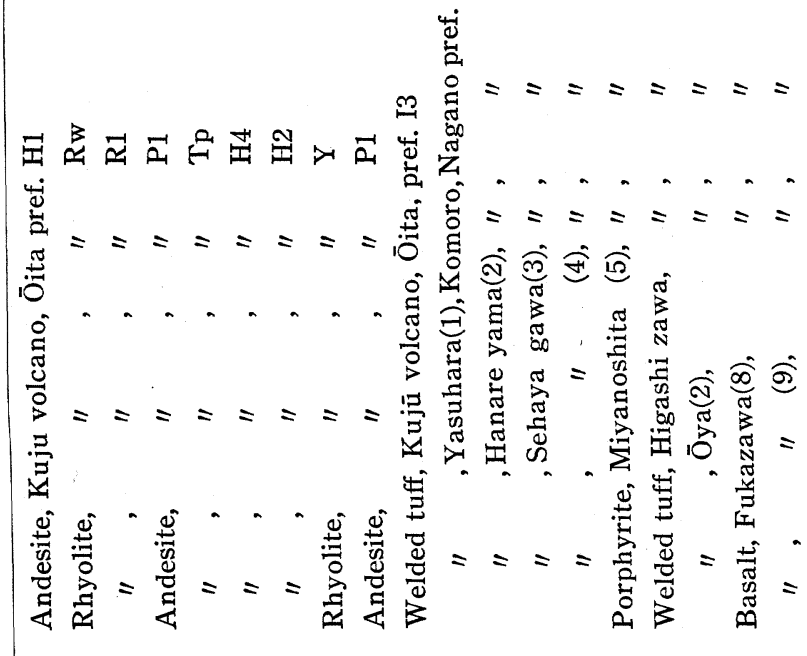 & 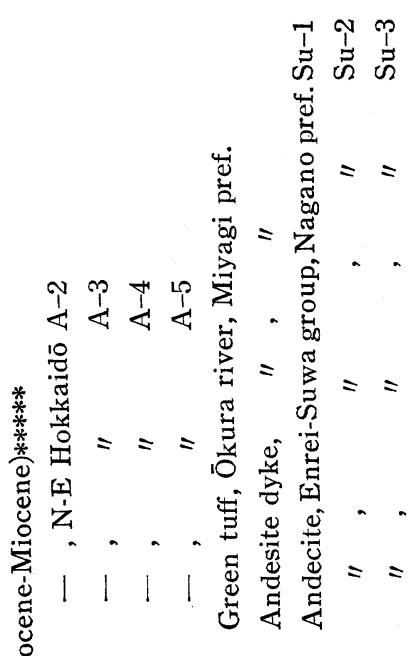 \\
\hline 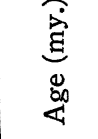 & $\begin{array}{llllllllllllllllll} & 1 & 1 & 1 & 1 & 1 & 1 & 1 & 1 & 1 & 1 & 1 & 1 & 1 & 1 & 1 & 1 & 1\end{array}$ & $\begin{array}{lllllllll}1 & 1 & 1 & 1 & 1 & 1 & 1 & 1 & 1\end{array}$ \\
\hline
\end{tabular}




\begin{tabular}{|c|c|c|c|c|}
\hline 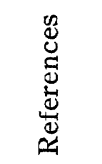 & 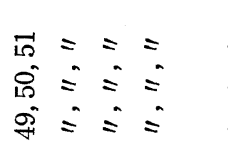 & 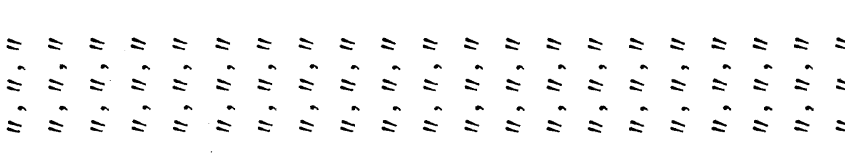 & & 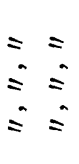 \\
\hline 离 & 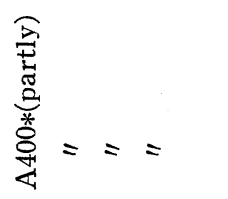 & 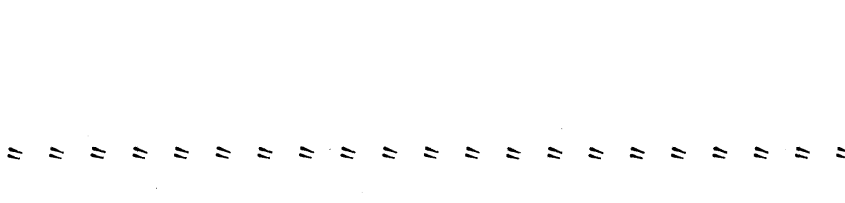 & & \\
\hline \multirow[t]{2}{*}{ \& } & 结 命 & 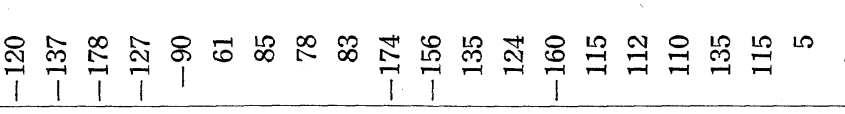 & & $\stackrel{\overrightarrow{0}}{1}$ \\
\hline & 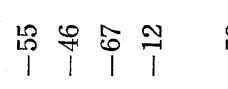 & 命 숭 & & ठ艹 \\
\hline 2 & $\sum 1 \mid \sum$ & $\begin{array}{llllllllllllllllllll} & 1 & \Sigma & 1 & 1 & 1 & 1 & 1 & 1 & 1 & 1 & 1 & \Sigma & 1 & 1 & 1 & \Sigma & 1 & 1 & 1\end{array}$ & & $1 \Sigma$ \\
\hline 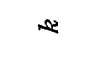 & 1111 & $\begin{array}{lllllllllllllllllllll}1 & 1 & 1 & 1 & 1 & 1 & 1 & 1 & 1 & 1 & 1 & 1 & 1 & 1 & 1 & 1 & 1 & 1 & 1 & 1 & 1\end{array}$ & 1 & 1 \\
\hline$\stackrel{8}{8}$ & 1111 & 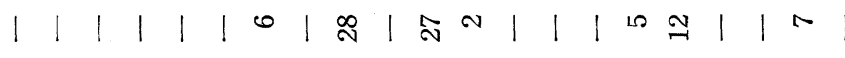 & 1 & \\
\hline$\theta$ & 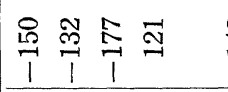 & ษ & & \\
\hline- & 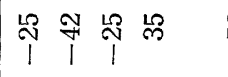 & 且 & & $\stackrel{\overbrace{}}{\rightarrow} \vec{m}$ \\
\hline$z$ & 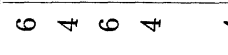 & 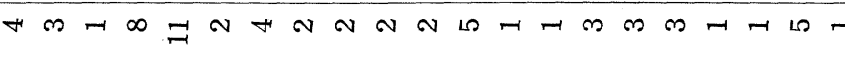 & & $\wedge \rightarrow$ \\
\hline 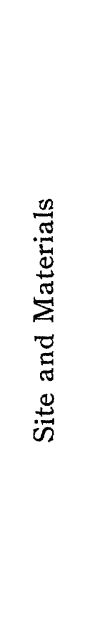 & 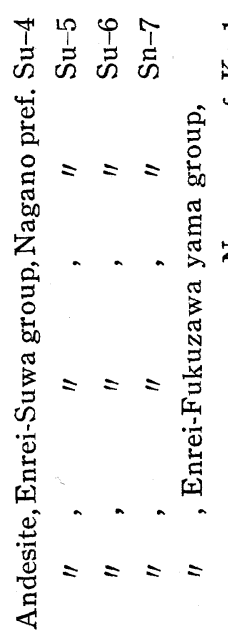 & 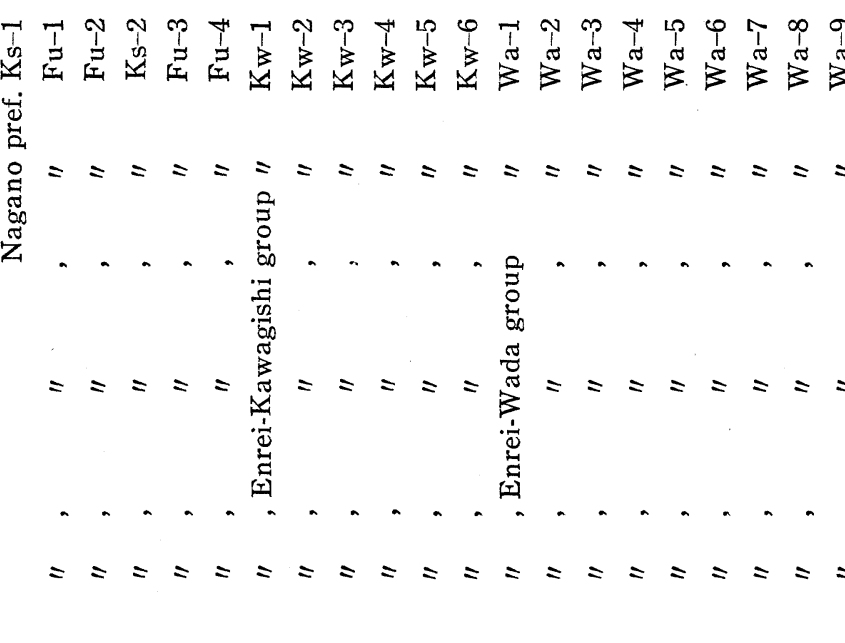 & 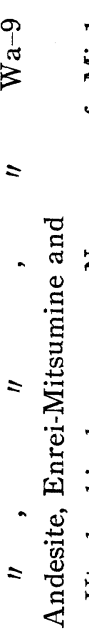 & 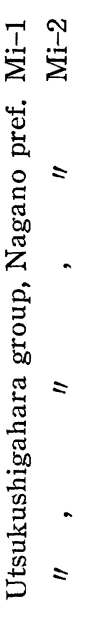 \\
\hline$\underset{\Delta}{\dot{\vec{E}}}$ & 11111 & $\begin{array}{llllllllllllllllllll}1 & 1 & 1 & 1 & 1 & 1 & 1 & 1 & 1 & 1 & 1 & 1 & 1 & 1 & 1 & 1 & 1 & 1 & 1 & 1\end{array}$ & 1 & \\
\hline
\end{tabular}




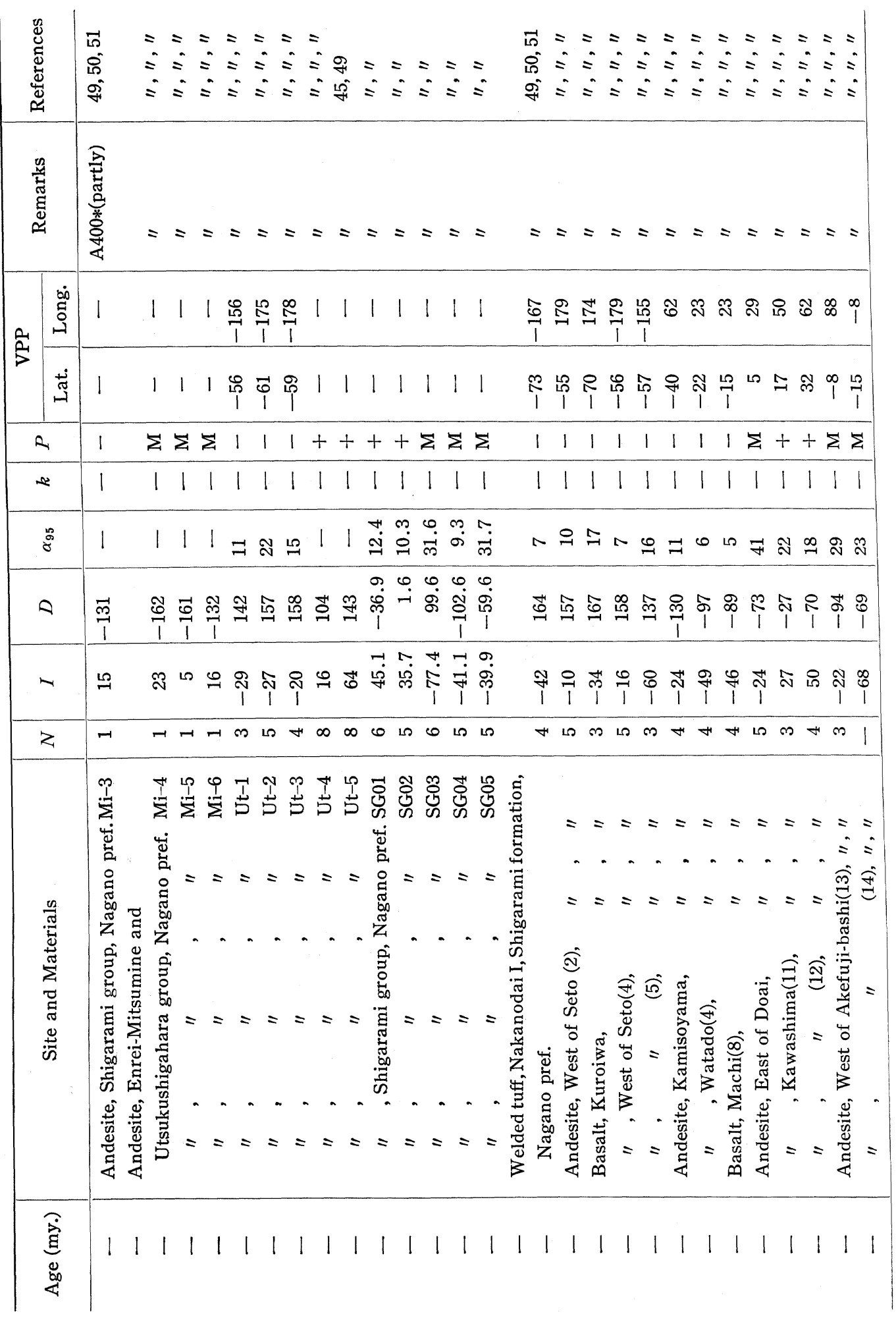




\begin{tabular}{|c|c|c|}
\hline 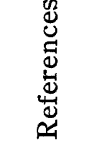 & 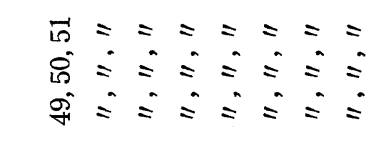 & 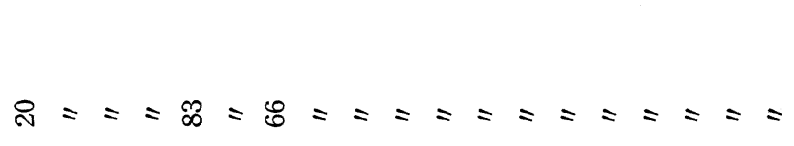 \\
\hline 䏰 & 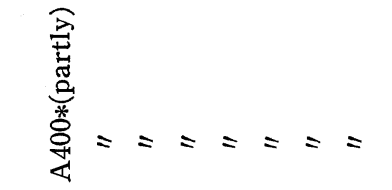 & $\dot{\varphi}==\frac{\sum_{n}}{\dot{\alpha}}=\frac{1}{\frac{1}{4}}==========$ \\
\hline \multirow{2}{*}{$\stackrel{a}{p_{1}}$} & 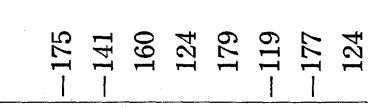 & 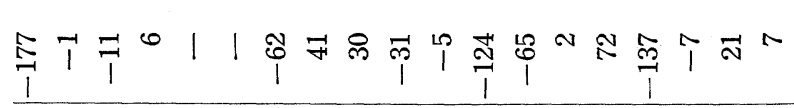 \\
\hline & ก & 同出里|| \\
\hline 2 & $+++\Sigma \Sigma+\Sigma \Sigma$ & $+\sum \sum \sum 1+11+++++++++++$ \\
\hline 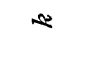 & $\begin{array}{llllllll}1 & 1 & 1 & 1 & 1 & 1 & 1 & 1\end{array}$ & $\begin{array}{lllllllllllllllllll}1 & 1 & 1 & 1 & 1 & 1 & 1 & 1 & 1 & 1 & 1 & 1 & 1 & 1 & 1 & 1 & 1 & 1 & 1\end{array}$ \\
\hline$\stackrel{8}{8}$ & 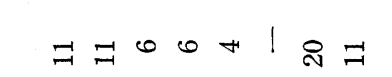 & 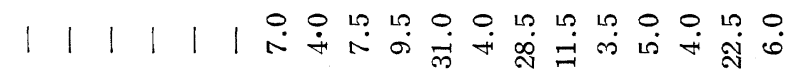 \\
\hline$\theta$ & จิ $\stackrel{\infty}{\rightarrow}$ 우 & の \\
\hline- & J゙ & 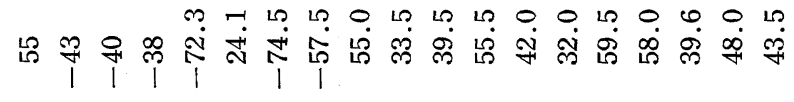 \\
\hline z & Lת m & L \\
\hline 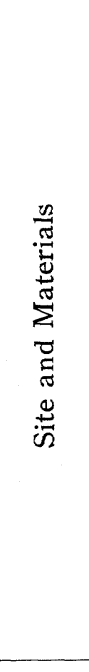 & 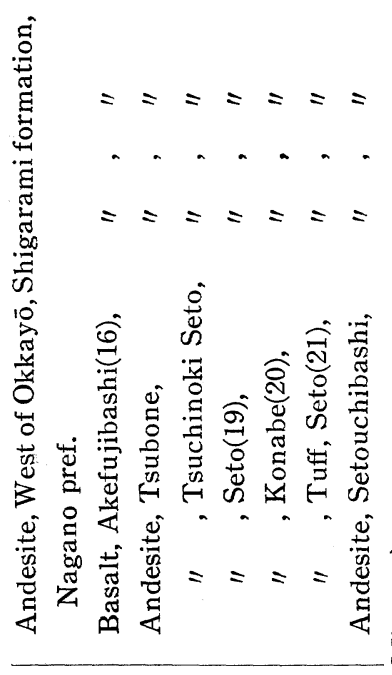 & 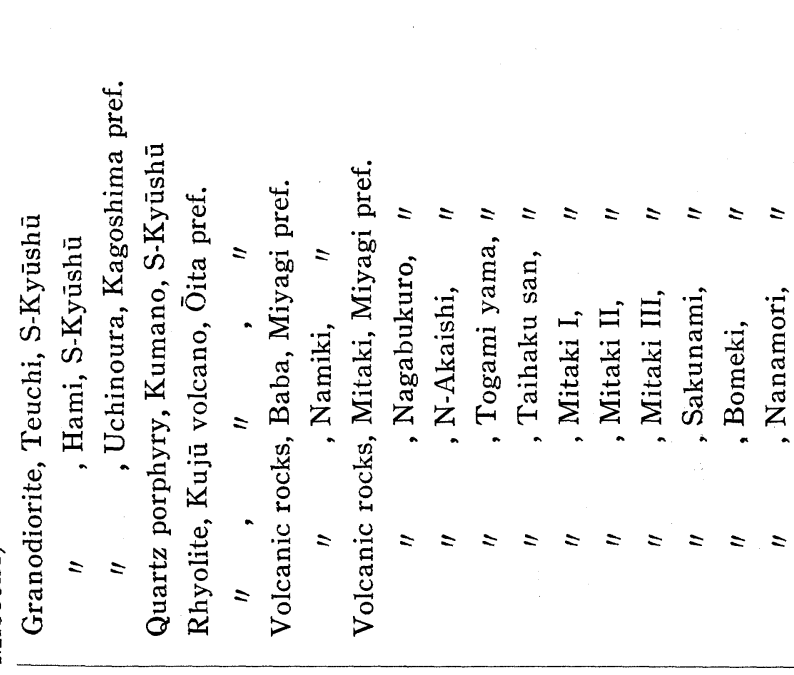 \\
\hline 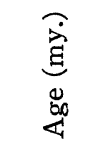 & 11111111 & $\underset{M}{\stackrel{H}{H}}$ \\
\hline
\end{tabular}




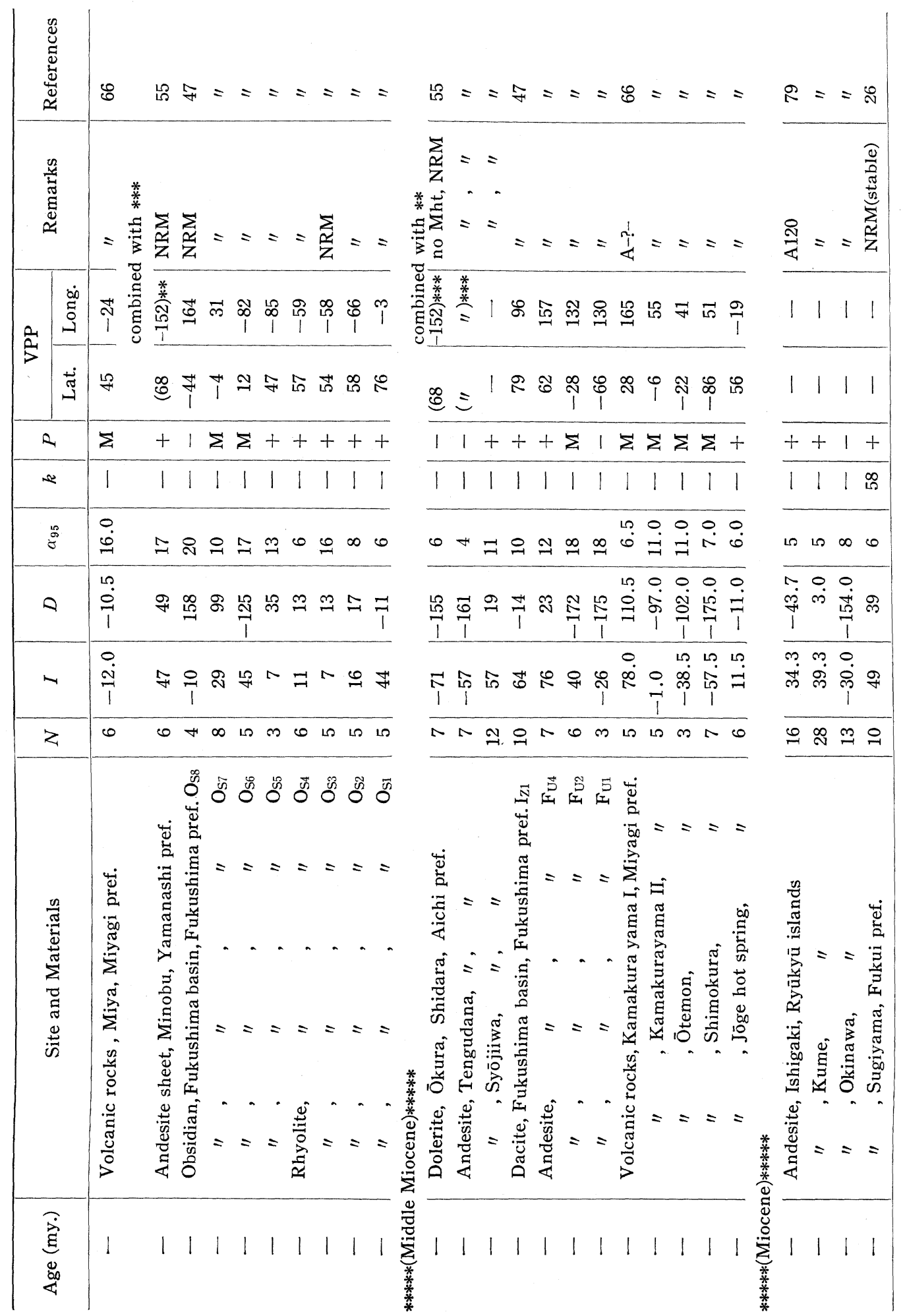




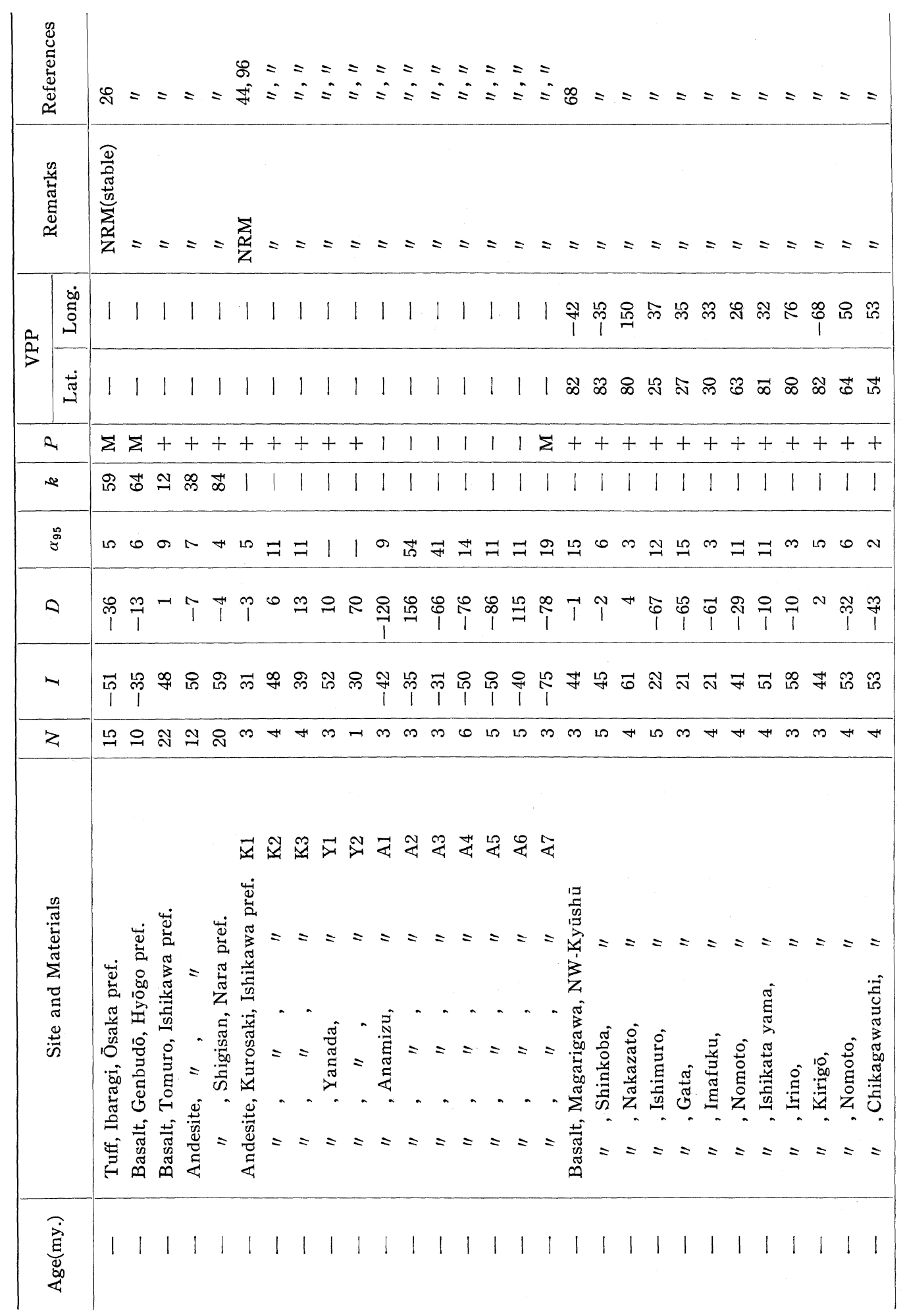




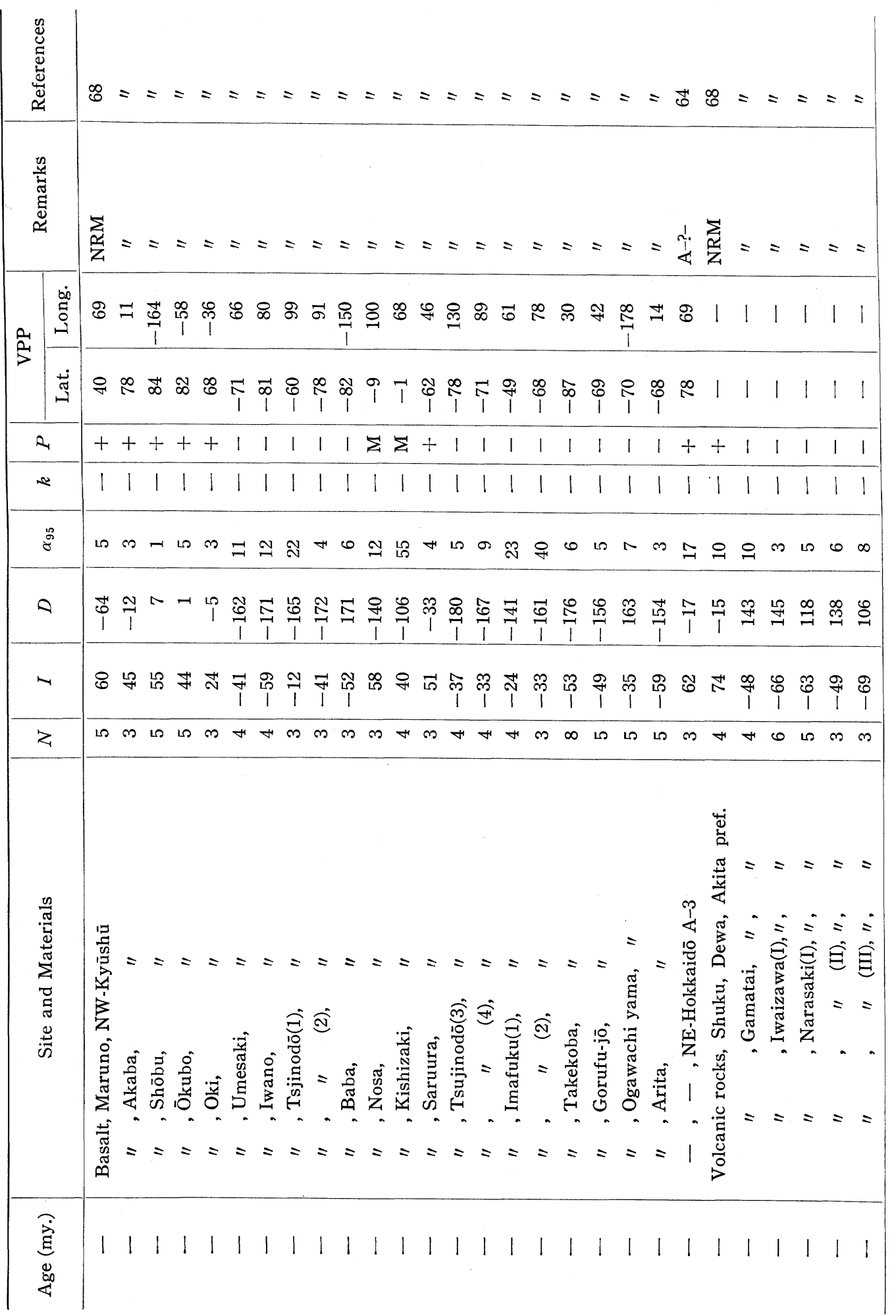




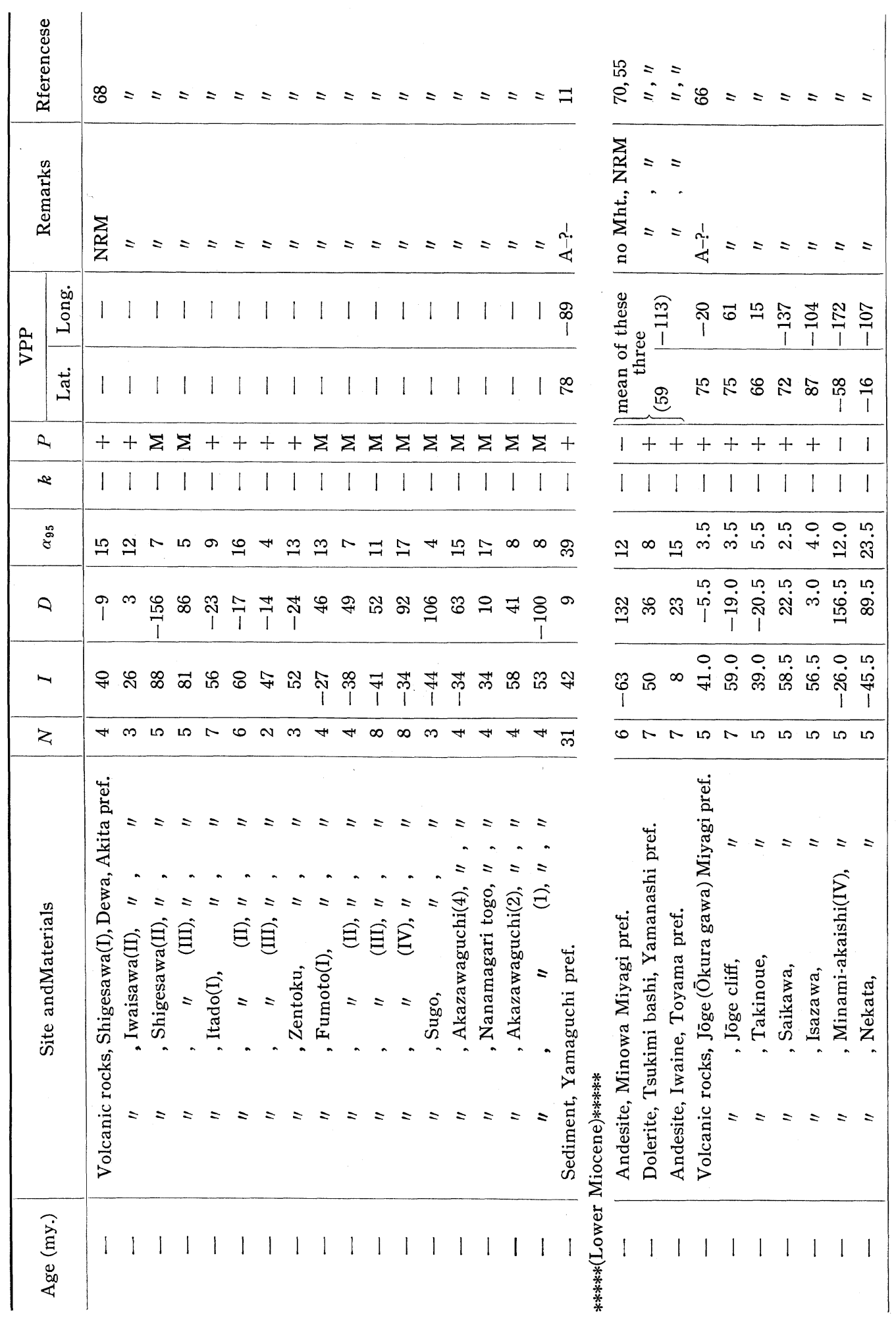




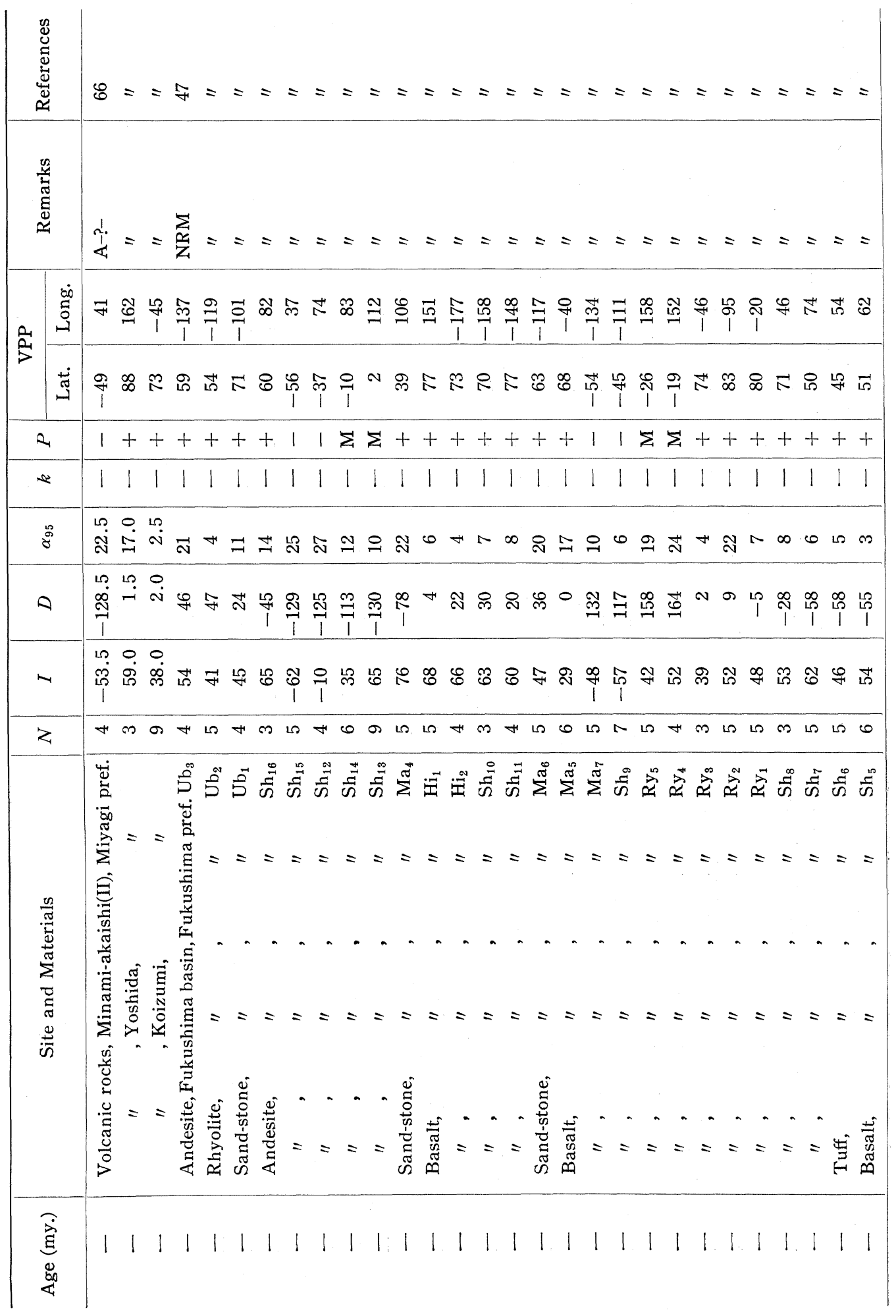




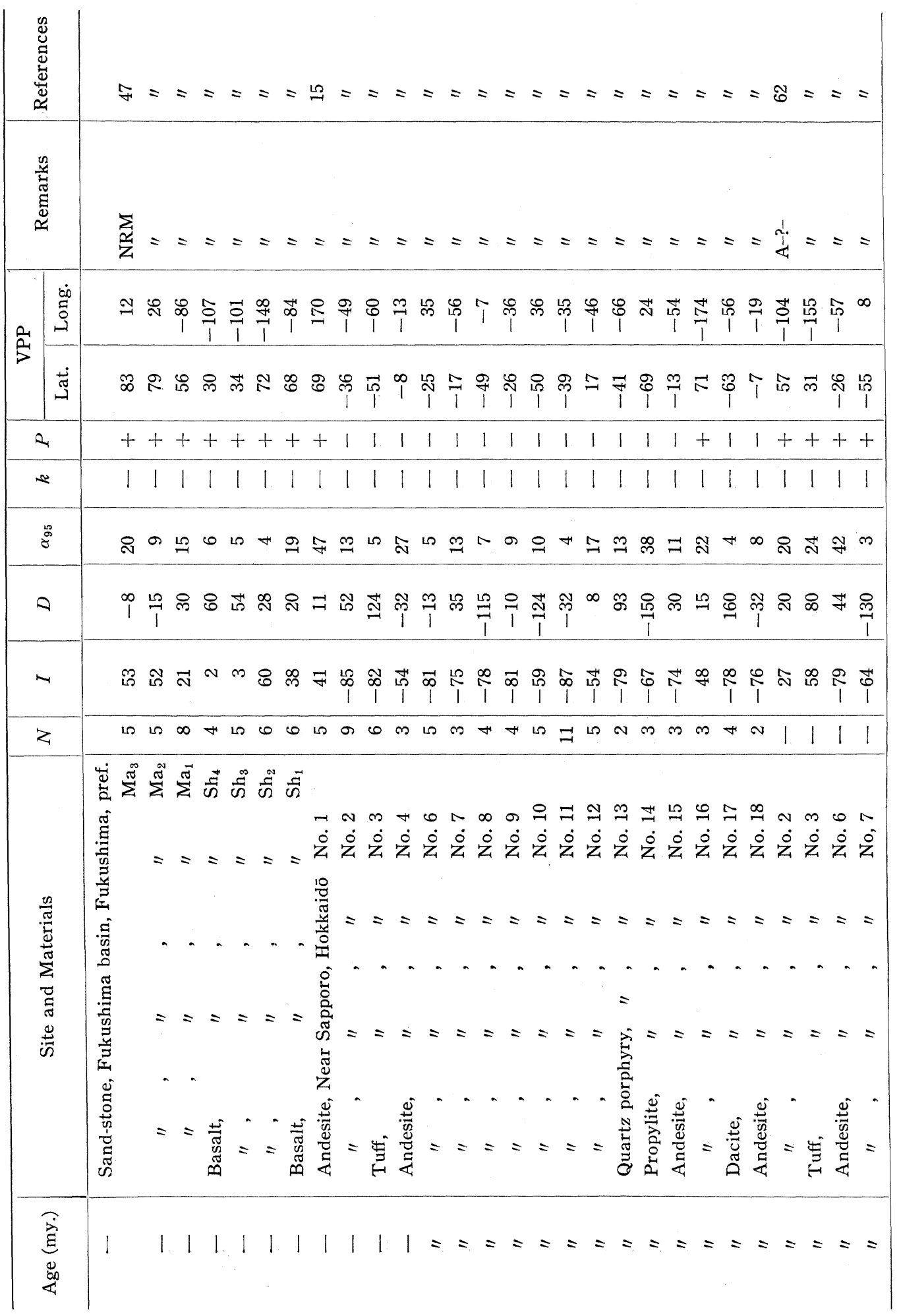




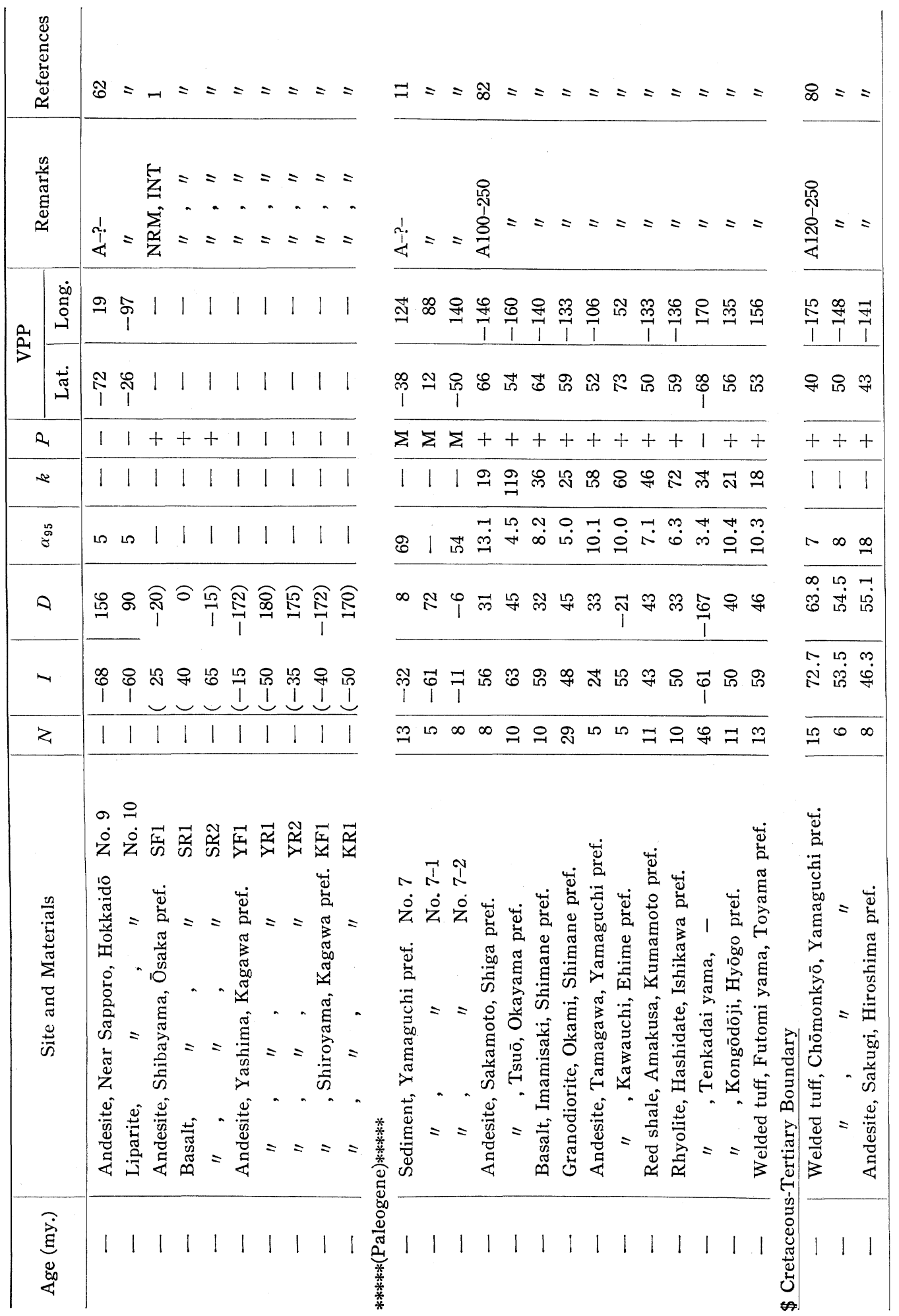




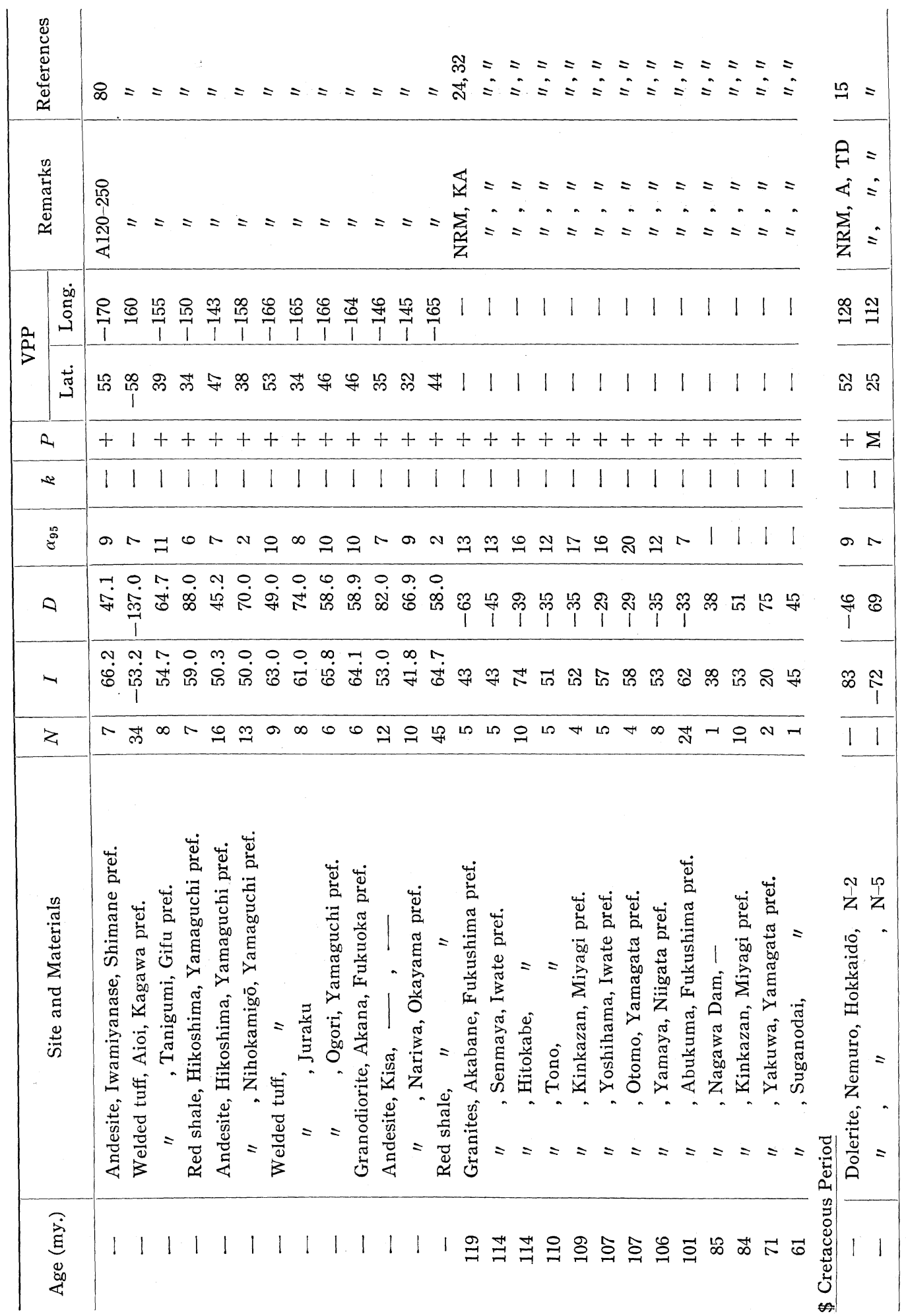




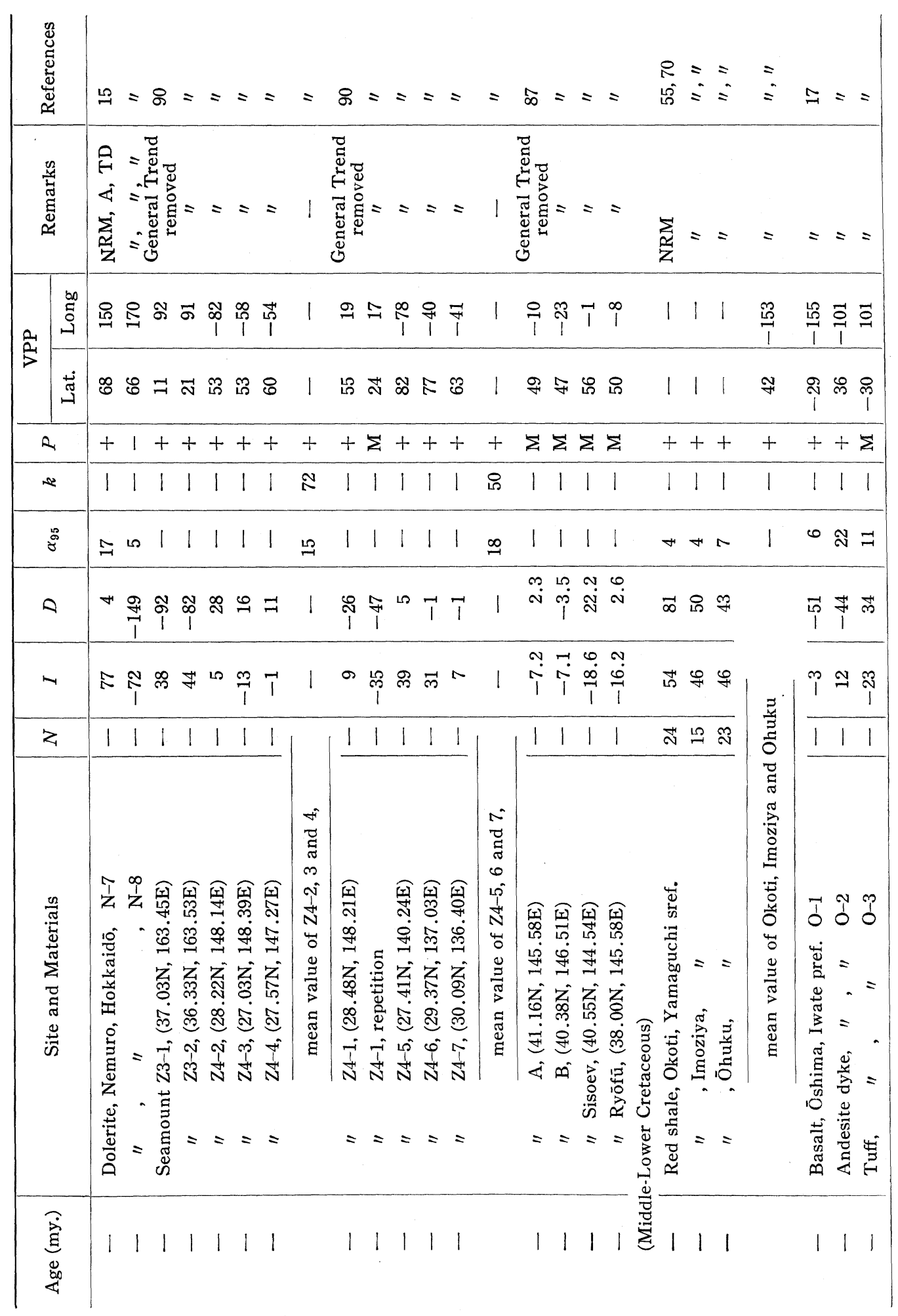




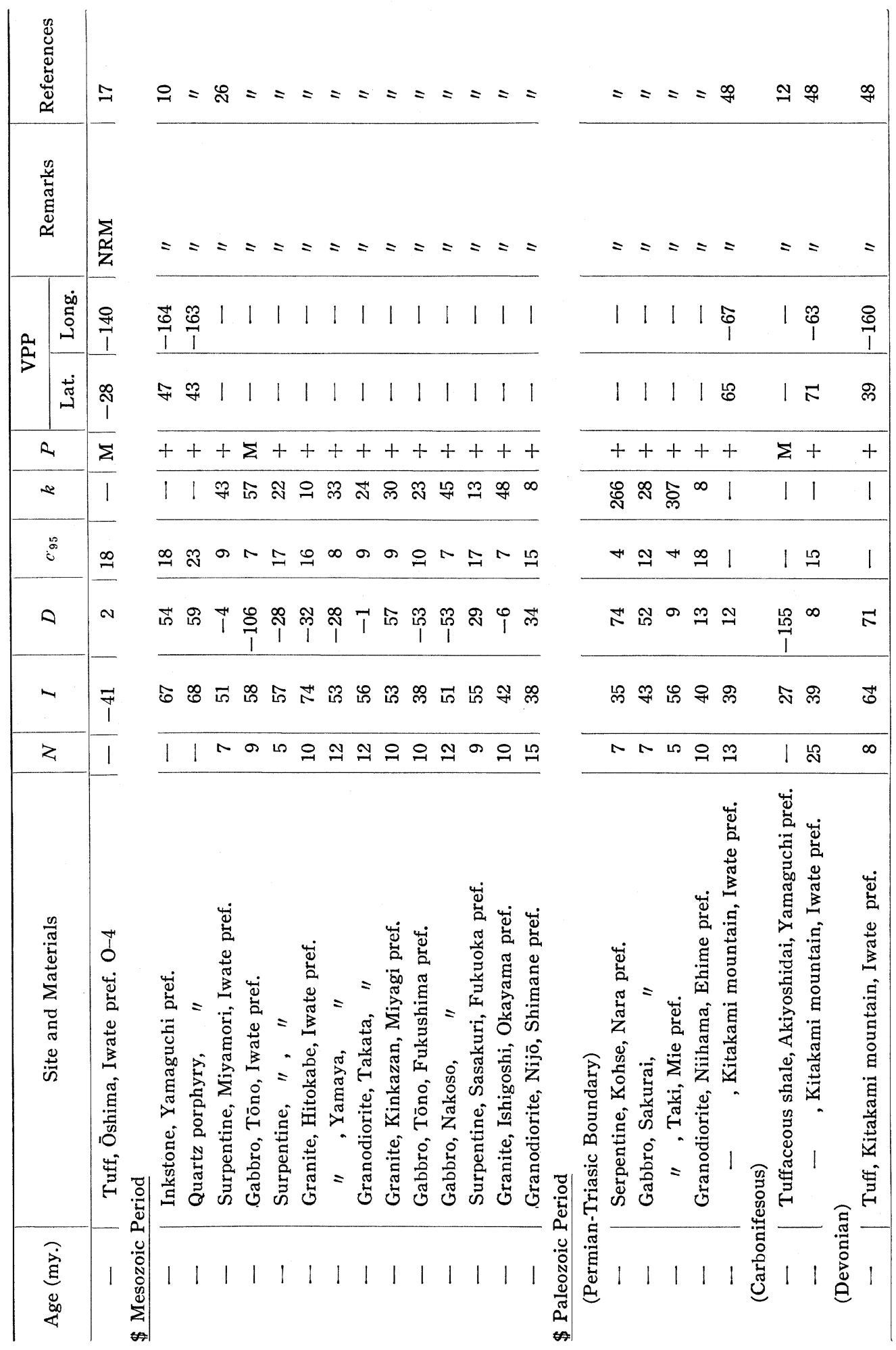




\section{Acknowledgment}

The author expresses his thanks to all of investigators referred in the table. They replied and gave willingly all the avairable data irrespective of published or unpublished till now upon inquiries of the present author. He owes greatly the present successful collection of data to all of them.

\section{Request from the Author}

The tables presented here may not be complete due to lack of data. It is considered that there must be much more data unpublished and/or over-looked by the present author. Therefore, for the completion of tables the present author would much appreciate if any data which are failed to appear here will be forwarded to him.

\section{References}

Aoki, M., Osaka shibayama no basaltic andesite yurai no seki-shoku dojō ni tsuite, $\mathrm{Ph} \mathrm{D}$. thesis, Osaka Furitsu Daigaku, 1969.

Aoki, Y., On the magnetization mechanism of ash fall tuffs, Thesis work, Master of Sci. Univ. Tokyo, 1970.

Aramaki, S.. Geology of the volcano Asama, Chigaku Dantai Kenkyukai. Senpō, 14, 1-45, 1968.

Asami, E., Reverse and normal magnetism of the basaltic lavas at Kawajiri-misaki, Japan, J. Geomag. Geoelectr., 6, 145-152, 1954.

Asami, E. and H. Domen, On the natural remanent magnetization of basalts in northern sea-side district of Nagato and sedimentary rocks in Susa-Tertiary layer, J. Sci., Yamaguchi Univ., 5, 1-20, 1954. (5)

Asami, E., A paleomagnetic consideration on the remanent magnetism of the basalt lavas at Kawajirimisaki, Japan, J. Geomag. Geoelectr., 8 146-155, 1956.

Asami, E. and H. Ito, Palaeomagnetic study on the early Pleistocene lavas in the Mishima and Oki islands. APRRMRGJ*, 60-66, 1964.

Asami, E., T. Kishi and A. Kurotani, Paleomagnetic study on the Pliocene lava near Mt. Daisen and the Miocene lava at Hamada area, APRPRGJ**, 90-94, 1967.

Domen, H., A short report on the remanent magnetization of Kawajiri-misaki (cape Kawajiri) basalts, Yamaguchi prefecture, Japan, APRRMRGJ. 145-149, 1965.

Domen, H., A preliminary study of the palaeomagnetism on the Mesozoic rocks of Yamaguchi prefecture, west Japan, APRRMRGJ, 151-151, 1965.

Domen, H., A paleomagnetic itudy of some tertiary and Quaternary rocks of Yamaguchi prefecture, Japan, J. Geophys. Res., 70, 425-432, 1965.

Domen, H., A memoir on the natural remanent magnetization of Kawajiri-Misaki basalts, Yamaguchi, west Japan, Bull, Fac, Education, Yamaguchi Univ., 15, 13-16, 1966.

Domen, H., An experimental study on the unstable remanent magnetization of rocks as a paleomagnetic fossil, Bull. Fac. Education. Yamaguchi Univ., 18, 1-31, 1969.

Domen, H. and M. Kawano, On the natural remanent magnetization of the tephra in diluvial deposits, Yamaguchi prefecture, southwest Japan, Yamaguchi Daigaku Kyoiku Gakubu Kenkyu Ronso, 18, 23-33, 1969.

Fujiwara, Y. and M. Nagase, Paleomagnetic studies of the Cretaceous rocks in the Nemuro peninsual, Hokkaido, Japan, Chikyu Kakaku, No. 79, 42-46, 1965.

Fujiwara, Y., Paleomagnetic studies on some Mesozoic rocks in Japan, J. Faculty of Sci., Hokkaido Univ., 8, No. 3, 293-300, 1966. 
Fujiwara, Y., Paleomagnetism of upper Carboniferous rocks in Akiyoshi province, s.w Honshu, Japan, ibid., 8, No. 4, 359-399, 1967.

Irving, E., Palaeomagnetism, John Wiley and Sone, 1964.

Isshiki, N., K. Nakamura, M. Hayakawa, K. Hirasawa, T. Yukutake, Y. Arai and B. Iwasaki, Structure of caldera of Oshima volcono, Izu, as revealed by drilling, Kazan 2-8, 61-106, 1963.

Ito, H., Palaeomagnetic study on Kyūshū outer zone granites, APRRMRGJ, 87-90, 1963.

\section{Kaneoka, I, M and M Ozima, M. Ayukawa and T Nagata, K-Ar ages and palaeomagnetic studies on} racks from the east coast of Lützow Holm bay, Antarctia, Antarctic Rec., No. 31, 12-20, 1968. (21)

Kaneoka. I., M. Ozima, and H. Kuno, Paleomagnetism and K-Ar ages of successive lava flows (4)-KAr ages of Usami volcano, Izu peninsula, Japan, to be published, J. Geomag. Geoelectr., 22, 1970. (22)

Kato, Y. and A. Takagi, Reverse remanent magnetism of dyke of basaltic andesite, J. Geomag. Geoelectr., 6, 206-207, 1954.

Kato, Y. and I. Muroi, Paleomagnetic studies of the Cretaceous granitic rocks in north-eastern Japan, APRRMRGI. 179-187, 1965.

Kawai, N., Magnetic polarization of Tertiary rocks in Japan, J. Geophys. Res. 56, 73-79, 1951.

Kawai, N., H. Ito and S. Kume, Defermation of the Japanese islands as inferred from rock magnetism, Geophys. J. R. astr. Soc., 6, 124-130, 1962.

Kawai, N., K. Hirooka, S. Sasajima, K. Yaskawa, H. Ito and S. Kune, Archaeomagnetic studies in southwestern Japan, APRRMRGJ. 39-43, 1964.

Kawai, N., K. Hirobka, S. Sasajima, K. Yasukawa, H. Ito and S. Kume, Archaeomagnetic studies in southwestern Japan, Ann. Geophys., 21, 574-578, 1965.

Kawai, N. and K. Hirooka, Wobbling motion of the geomagnetic dipole field in historic time during these 2,000 years, J. Geomag. Geoelectr., 19, 217-227, 1967.

Kawai, N., K. Hirooka and K. Tokieda, A vibration of geomagnetic axis around the geographic north pole in the historic time, Earth Planet. Sic. Lett., 3, 48-50, 1967.

Kawai, N., K. Hirooka, K. Tokieda and T. Kishi, Archaeo-secular variation of the geomagnetic field in Japan, $A P R P R G J, 81-85,1967$ and private communication.

Kawai, N., K. Hirooka and T. Nakajima, Paleomagnetic and potassium-argon age informations supporting Cretaceous-Tertiary hypothetic bend of the main island Japan, Paleogegraphy, Paleoclimatol., Paleoecol., 6, 277-282, 1969.

Kinoshita, H., K. Kobayashi, M. Kono, E. Larson, T. Nagata, M. Ozima and D. Strangway, Paleomagnetism of Pliocene basalts from southwestern United States, APRRMRGJ, 111-132, 1965.

Kinoshita, H., and M. Ohnaka, Magnetism of rocks from a lava dome, Mt. Minakami - Application to earthquake prediction (1)-Kazan, 2-13, 1-11, 1968.

Kitazawa, K. and K. Kobayashi, Intensity variation of the geomagnetic field during the past 4,000 years in south America, J. Geomage. Geoelectr.. 20, 7-19, 1968.

Kitazawa, K., Chikyu jikai kyodo no einen henka (Secular variation of the geomagnetic field intensity), Nihon Butsuri Gakukaishi, 24, 25-27, 1969 and private communication. (See addendum)

Kobayashi, K., Field intensity in the Precambrian period, APRPRGJ, 105-111, 1967.

Kono, M., H. Kinoshita, T. Nagata and H. Kuno, Palaeomagnetic study of the lava flows of the Hakone volcanoes, APRRMRGJ, 54-59,1964.

Kono, M. and T. Nagata, Intensity of the geomagnetic field during a reversed polarity, Nature, 212, 274-275, 1966.

Kono, N., Studies of the earth's magnetic field during Tertiary and Quaternary by paleomagnetic methods, Thesis work, Univ. Tokyo, 1966.

Kono, M., K. Kobayashi, M. Ozima, H. Kinoshita, T. Nagata, E. Larson and D. Strangway, Paleomagnetism of Pliocene basalts from the southwestern U.S.A., J. Geomag. Geoelectr., 19, 357-375, 1967. 
Kono, M., Paleomagnetism of Pleistocene Usami volcano, Izu peninsula, Japan -Intensity of the earth's magnetic field in geological time II, J. Geomag. Geoelectr., 20, 353-366, 1968.

Kono, M. and T. Nagata, Intensity of the earth's magnetic field in geological time I, Late Pliocene in the southwestern U.S.A. J. Geomag. Geoelectr., 20, 211-220, 1968.

Kono, Y., K. Nakagawa, O. Hasukawa and H. Yashiki. Physical properties and palaeomagnetic studies of andesite at the Noto peninsula, Japan. Chikyu Kagaku, 23, 243-251, 1969.

Kono, M., Intensity of the earth's magnetic field in Pliocene and Pleistocene, $\mathrm{Ph}$. D. thesis, Univ. Tokyo, 1970.

Maenaka, K., S. Ishida and T. Yokoyama, Preliminary result on the paleomagnetic chronology of the Osaka group, APRPRGJ, 86-94, 1967.

Manabe, K., Results of paleomagnetic studies of miocene volcanic rocks in the Fukushima district, northeast Honshū, Japan, J. Geol. Soc. Japan. 73, 325-336, 1967.

Minato, M. and Y. Fujiwara, Magnetic pole pesitions determined by the Japanese Palaeozoic rocks, Chikyu Kagaku, No. 78, 21-22. 1965.

Momose. K., Paleomagnetic researches of the Pliocene volcanic rocks in central Japan (1), J. Geomag. Geoelectr., 10, 12-19, 1958-59.

Momose, K., K. Kobayashi and T. Yamada, Paleomagnetic and geologic researches for the volcanic rocks around lake Suwa. -Palaeomagnetic researches for the Pliocene volcanic rocks in central Japan (2)-, Bull. Earthquake Res. Inst., 37, 433-481, 1959.

Momose, K., Studies on the variations of the earth's magnetic field during Pliocen time, Bull. Earthquake Res. Inst., 41, 487-534, 1963.

Momose, K., K. Kobayashi, K. Tsuboi and M. Tanaka, Archaeomagnetism during the old Tomb and Nara period, APRRMRGJ, 33-38, 1964.

Momose. K. and K. Kobayashi, Magnetic direction of the baked earth at the earliest Jomon cultural remains in Kita-Aiki mura, Nagano prefecture-A preliminary report, APRRMRGJ, 101-104, 1965. (53)

Nagata, T., S. Akimoto, S. Uyeda, Y. Shimizu, M. Ozima, K. Kobayashi and H. Kuno, Paleomagnetic studies on a Quaternary volcanic region in Japan, J. Geomag. Geoelectr., 9, 23-41, 1957.

Nagata, T., S. Akimoto Y. Shimizu K. Kobayashi and H. Kuno, Palaeomagnetic studies on Tertiary and Cretaceous rocks in Japan, Proc. Japan Acad., 35, 378-383. 1959.

Nagata, T. and Y. Shimizu, Paleomagnetic studies on Precambrian gneiss of Ongul island, Antarctica, Nature, 184, 1472, 1959.

Nagata, T. and M. Yama-ai, Paleomagnetic studies on rocks on the coast of Lützw Holm bay, Antarctic Rec., No. 11, 945-947, 1961.

Nagata, T., Rock magnetism, Revis, Ed., Maruzen. Tokyo. 1961.

Nagata, T., Y. Arai and K. Momose, Secular variation of the geomagnetic total force during the last 5,000 years. J. Geophys. Res., 68, 5277-5281, 1963.

Nagata, T,, M. Ozima. Y. Syono, M. Ozima, Y. Arai, H. Kinoshita and H. Kuno, Preliminary report on palaeomagnetic study of the lava fiows of the Hakone volcanoes, APRRMRGJ.101-105, 1963. (60)

Nakagawa, H., N. Niitsuma and I. Hayasaka, Late Cenozoic geomagnetic chronology of the Bōsō peninsula, Chishitsugaku Zasshi, 75, 267-280. 1969. (See addendum)

Nishida, Y. and I. Yokoyama, Palaeomagnetic study in south-western part of Hokkaido, APRRMRGJ, 163-171, 1965.

Nishida, Y., Paleomagnetic study around Sapporo in Hokkaido (1st papar), Geophys. Bull. Hokkaido Univ., 15, 60-78, 1966.

Nishida, Y., Paleomagnetic study in northern part of Hokkaido, APRPRGJ, 95-98, 1967.

Nishiyama, Y., Paleomagnetic study of the volcanic rocks in northeast part of Yatsugatake volcanic chain, Chikyu Kagaku No. 82, 1-8, 1966.

Nomura, S., Paleomagnetic studies on the Neogene volcanic rocks from the Sendai district, northeast 
Honshy, Japan, Chikyu Kagaku, Nos. 67 and 68, 30-39, 1963.

Nomura, S., Upper Pliocene naishi lower diluvium no gyakutenjika shite iru ganseki ni tsuite, Hiramatsu Yoshinao sensei taishoku kinen ronbun-shū, 1967.

Nomura, S., On the properties of the paleomagnetic polarity of the Neogene and Quaternary rocks in Japan, Gumma Daigaku Kyoyobu Kiyo, 1, 11-35, 1967.

Ohnaka, M.. Stability of remanent magnetization of rocks under compression, J. Geomag, Geoelectr., 21, 495-505, 1969.

Ozima, M,. Review of paleomagnetic study at Geophysical Institute University of Tokoy, Chikyu Kagaku, No. 65, 20-23, 1963.

Ozima, M., M. Kono, I. Kaneoka, H. Kinoshita, K. Kobayashi, T. Nagata, E.E. Larson and D. Strangway, Paleomagnetism and potassium-argon ages of some volcanic rocks from the Rio-Grande gorge, New Mexico, J. Geophys. Res., 72, 2615-2621, 1967.

Ozima, M., I. Kaneoka, M. Kinoshita, K. Kobayashi, M. Ohnaka, T. Nagata and H. Kurasawa, Paleomagnetism and K-Ar ages of successive lava flows (2) Kita-Matsuura basalts, Kyūshu, Japan APRPRGJ. 48-53, 1967.

Ozima, M., I, Kaneoka, M. Kono, H. Kinoshita, K. Kobayashi, M. Ohnaka, T. Nagata and S. Aramaki, $\mathrm{K}$-Ar ages and paleomagnetism of successive lava flows (3) Hanamagari and Kirizumi andesites, Gumma prefecture, Japan, APRPRGJ. 54-62, 1967.

Ozima, M., I. Kaneoka, M. Kono, H. Kinoshita, K. Kobayashi, M. Ohnaka, T. Nagata and H. Kurasawa, Paleomagnetism and K-Ar ages of successive lave flows (2)-Kita-Matsuura basalt, Kyūsaū, Japan-, J. Geomag. Geoelectr., 20. 85-92, 1968.

Ozima, M., I. Kaneoka, M. Kono, H. Kinoshita, K. Kobayashi, M. Ohnaka, T. Nagata and S. Aramaki, Paleomagnetism and K-Ar ages of successive lava flows (3)-Hanamagari and Kirizumi andesites, Gumma prefecture, Japan-, J. Geomag. Geoelectr. 20. 101-105, 1968.

Rikitake, T., Electromagnetism and the earth's interior, Elsevier, Amsterdam. 1966.

Sasajima, S., On the causation of the NRM in plutonic rocks, APRRMRGJ, 77-80, 1963.

Sasajima, S. and K. Maenaka, Intensity studies of the geomagnetic field for the past 2,000 pears in west Japan, APRRMRGJ, 44-48, 1964.

Sasajima, S. ond M. Shimada, The palaeomagnetic study upon the geotectonics of the Ryūkyū islands, APRRMRGJ, 67-70, 1964.

Sasajima, S. and M. Shimada, Drift of the Honshū island during Cretaceous and Paleogene as inferred from paleomagnetic studies of southwest Japan, APRRMRGJ, 133-144, 1965.

Sasajima, S., Geomagnetic secular variation revealed in the baked earths in west Japan (part 2), Change of the field intensity, J. Geomag. Geoelectr., 17, 413-416, 1965.

Sasajima, S., J. Nishida and M. Shimada, Paleomagnetic Evidence of a drift of the Japanese main island during the Paleogene period, Earth Planet. Sci. Lett., 5, 135-141, 1968.

Sasajima, S. and K. Maenaka, Variation of the geomagnetic field intensity since the late Miocene, $J$. Geophys. Res. 74, 1037-1044, 1969.

Smith, P., The intensity of the ancient geomagnetic field: A review and analysis, Geophys. J.R. astr. Soc., 12, 321-362, 1967.

Takeshita, H., Y. Sato and K. Momose, Paleomagnetisms and volcanic geology of the Shigarami formation, Chikyu Kagaku, No. 49, 26-36, 1960.

Uyeda, S., T. Sato, M. Yasui and Y. Hagiwara, Report on geomagnetic survey in the northwestern pacific during JEDS-VI, JEDS-VII and JEDS-VIII cruises, Bull. Earthquake Res. Inst., 42, 557-570, 1964.

Uyeda, S. and M. Richards, Magnetization of four pacific seamounts near Japanese islands, APRRMRGJ, 189-223, 1965.

Uyeda, S. and M. Richards, Magnetization of four seamounts near Japanese islands, Bull, Earthquake 
Res. Inst., 44. 179-213,'1966.

Uyeda, S. and V. Vacquier, Paleomagnetism of nine seamounts in the western pacific and three volcanoes in Japan, APRPRGJ, 28-34, 1967,

Vacquier, V. and S. Uyeda, Paleomagnetism of nine seamounts in the western pacific and of three volcanoes in Japan, Bull. Earthquake Res. Inst., 45, 815-843, 1967.

Watanabe, N., Secular variation in the direction of geomagnetism as the standard scale for geomagnetochronology in Japan, Nature 182, 383-384, 1958.

Watanabe, N., The direction of remanent magnetism of baked earth and its application to chronology for anthropology and archaeology in Japan, J. Fac. Sic. Univ. Tokyo, Sec. V. Vol. II, 1-88, 1959. (92)

Watanabe, N., Some measurements of the direction of remanent magnetism of baked earth from archaeological sites in Peru, APRRMRGJ, 30-32, 1964.

Watanabe, N., Magnetic dating for anthropology and archaeology in Japan: A review of recent advance, Dai-yonki Kenkyu, 6, 230-238, 1967.

Watanabe, N. and M. Suzuki, Fission track dating of archaeological glass material from Japan, Nature, 222, 1057-1058, 1969.

Yashiki, H. Noto-hanto ni sansurn kazanganrui no kochijikigakuteki kenkyu, Kanazawa Daigaku Rigakubu Chigaku Kyoshitsu, 1968.

Yokoyama, I., Y. Nishida and T. Mori, Preliminary report on palaeomagnetic study of south-western part of Hokkaido APRRMRGJ, 49-53, 1964.

Yukutake, T., Archaeomagnetic study on volcanic rocks in Oshima island, Japan, Bull. Earthquake Res. Inst., 39, 467-476, 1961.

Yukutake, T., The westward drift of the magnetic field of the earth, Bull. Earthquake Res. Inst., 40, $1-65,1962$.

Yukutake, T., K. Horai and K. Nakamura, Magnetization of tuff in Oshima island and its application to archaeomagnetism and volcanology, APRRMRGJ, 81-86, 1963.

Yukutake. T., M. Sawada and T. Yabu, Magnetization of ash-fall tuffs of Oshima volcano, Izu, I. Magnetization of ash-fall tuffs J. Geomag. Geoelectr., 16, 178-182, 1964.

Yukutake, T., K. Nakamura aud K. Horal, Magnetization of ash-fall tuffs of Oshima volcano, Izu, II. Application to archaeo-magnetism and volcanology, J. Geomag. Geoelectr., 16, 183-193, 1964. (102)

\section{Addendum}

Thorough paleomagnetic studies on normal and reverse successions in sedimentary rocks of Tertiary and Quaternary periods are referred to in a paper; in Nakagawa, H., N. Niitsuma and I. Hayashi, Late Cenozoic geomagnetic chronology of the Bōsō peninsula, Chishitsugaku Zasshi, 75, 267-280, 1969. More detailed study on the normal reverse boundaries of Tertiary and Quaternary periods is referred to in a paper; Ito, H., Polarity transitions of the geomagnetic field deduced from the natural remanent magnetization of Tertiary Quaternary rocks in southwest Japan, J. Geomag. Geoelectr. 22, 273-290, 1970.

Supplement to (36); Kitazawa, K., Intensity of the geomagnetic field in Japan for the past 10,000 years, J. Geophys. Res., 75, 7403-7412, 1970.

\footnotetext{
* Annual Progress Report of the Rock Magnetism Research Group in Japan.

** Annual Progress Report of Palaeogeophysics Research Group in Japan.
} 